\title{
Protestzwitschern. Wie Twitter-Netzwerke zur Eskalation der G20-Proteste in Hamburg 2017 beitrugen ${ }^{1}$
}

\author{
Eddie HARTMANN² ${ }^{2}$ Felix LANG $^{3}$
}

Heidelberg, Potsdam

\begin{abstract}
Der Beitrag fragt, inwiefern das soziale Medium Twitter zur gewaltsamen Eskalation der G20-Proteste in Hamburg 2017 beigetragen hat. Die These dazu lautet, dass sich parallel zur Eskalation des Protestgeschehens auf den Straßen Hamburgs eine antagonistische Diskursdynamik in Twitter ereignet, die eine sich selbst verstärkende Spirale aus Solidarisierung und Feindbildkonstruktion begünstigt. Die vorliegende Analyse untermauert somit die inzwischen sowohl in der Protest- und Bewegungsforschung als auch in der Gewaltsoziologie gängige Annahme, dass eskalierende Konfliktverläufe notwendig mit Transformationen kognitiver Wahrnehmungs- und Deutungsmuster einhergehen, die durch ihren polarisierenden Effekt zu einem zentralen Bestandteil der Verursachungsstruktur gewaltsamer Konflikte werden.
\end{abstract}

Schlüsselwörter: Gewalt, Protest, Eskalation, Soziale Medien, Twitter, Netzwerkanalyse

\section{Einleitung}

Die Ereignisse rund um den G20-Gipfel im Juli 2017 in Hamburg haben eine Menge verbrannte Erde hinterlassen. In Erinnerung geblieben ist nicht etwa das im Vorfeld des Gipfels von der

\footnotetext{
${ }^{1}$ Der folgende Beitrag stützt sich auf zentrale Ergebnisse des Projekts Mapping \#NoG20, das Ende 2017 mit Unterstützung zahlreicher Stiftungen ins Leben gerufen wurde, darunter die Hamburger Stiftung zur Förderung von Wissenschaft und Kultur und die Zeit-Stiftung. Koordiniert vom Institut für Protest- und Bewegungsforschung (ipb), dem Zentrum Technik und Gesellschaft der Technischen Universität Berlin (ZTG) und dem Hamburger Institut für Sozialforschung (HIS) wurde das Projekt durch ein Netzwerk von mehr als 20 Wissenschaftlerinnen und Wissenschaftlern aus der Protest-, Polizei- und Gewaltforschung getragen. Siehe hierzu der Bericht des Forschungsprojekts Mapping \#NoG20: Eskalation. Dynamiken der Gewalt im Kontext der G20-Proteste in Hamburg 2017 (Malthaner et al. 2018).

${ }^{2}$ Dr. habil. Eddie Hartmann vertritt aktuell die Professur für Politische Soziologie am Max-Weber-Institut für Soziologie der Universität Heidelberg (eddie.hartmann@mwi.uni-heidelberg.de).

${ }^{3}$ Felix Lang, MA, Doktorand und Stipendiat der Graduiertenförderung des Landes Brandenburg (felix.lang@uni-potsdam.de).
} 
Hamburger Politik angekündigte Festival der Demokratie, sondern Bilder von Zerstörung, Gewalt und sozialen Verwerfungen. Bis heute sind die Ereignisse von Hamburg Gegenstand einer polarisierten Debatte, die vor allem von einseitigen Schuldzuschreibungen geprägt ist (Sommer/Teune 2019). Die Frontlinie zwischen den dominierenden Stellungnahmen verläuft in der Regel zwischen einer Diskreditierung der Protestseite einerseits, wonach die Gewalt von der radikalen Linken gezielt vorbereitet und von Teilen des gemäßigten Protestspektrums zumindest gebilligt, teilweise gar befürwortet worden sei; und der politischen Rehabilitierung des Protests andererseits, indem behauptet wird, der Hamburger Senat und die Polizei hätten mit der Kriminalisierung von Protest, mit der Einschränkung von Grundrechten und mit illegaler Polizeigewalt die Ausschreitungen billigend in Kauf genommen, wenn nicht herbeigeführt. Daraufhin habe nicht nur der gewaltsame Protest, sondern der Protest insgesamt seine politische Legitimität in den Augen großer Teile der Öffentlichkeit weitgehend eingebüßt (daher der Versuch der politischen Rehabilitierung).

Sowohl die Diskreditierungs- als auch die Rehabilitierungsthese sind politisch eingefärbte Deutungen, die, an wissenschaftlichen Ansprüchen gemessen, das kollektive Gewaltphänomen im Kontext der G20-Proteste nur unzureichend zu beschreiben erlauben. Beide Deutungsangebote führen unweigerlich zu kausalen Kurzschlüssen. Statt die eigendynamischen Anteile und das Eskalationspotenzial kollektiver Gewaltphänomene dieser Art gebührend zu berücksichtigen, rücken sie entweder stabile Handlungsmotive individueller Akteure oder das strategische Kalkül von Organisationen und sozialen Bewegungen in den Mittelpunkt der Erklärung. Mit der passenden soziologischen Intuition für das Konfliktgeschehen sprach daher der Direktor des Bielefelder Instituts für Interdisziplinäre Konflikt- und Gewaltforschung, Andreas Zick, in einem Interview mit den Tagesthemen vom 10.7.2017 davon, dass ideologische Motive für das Ausmaß der gewaltsamen Ausschreitungen letztlich keine tragende Rolle gespielt hätten (siehe auch Deutschlandfunk 2017). Stattdessen, so Zick, sei die Gewalt selbst zum verbindenden Element zwischen den Gewaltakteuren geworden. In diesem Sinne sprach Zick unter anderem von einer Gewaltmasse - ein Begriff, der in der deutschsprachigen Diskussion über kollektive Gewaltphänomene und ihre situativen Dynamiken auch dank eines Sammelbandes von Axel T. Paul und Benjamin Schwalb aus dem Jahr 2015 wieder stärker in den Fokus gerückt ist. Unter einer Gewaltmasse verstehen die Autoren „nicht-organisierte, darum jedoch nicht unbedingt unstrukturierte Kollektive kopräsenter Akteure, die gemeinschaftlich, deswegen jedoch nicht planvoll, physische Gewalt gegenüber Dritten ausüben“" (Paul/Schwalb 2015: 10). Der von Andreas Zick ins Spiel gebrachten Interpretation der Ereignisse schließen wir uns im Folgenden grundsätzlich an. Dabei möchten wir der Frage nachgehen, inwiefern das soziale Medium Twitter dazu beigetragen hat, dass die G20-Proteste in Hamburg einen derartigen Verlauf genommen haben. Genauer: Welchen spezifischen Anteil tragen Twitter-Netzwerke an der Emergenz jener Gewaltmasse, von der Andreas Zick in seinem Interview gesprochen hat?

Die Fragestellung dieses Beitrags zielt somit auf gewaltgenerierende Gruppenprozesse ab, die mittlerweile maßgeblich vom Gebrauch moderner elektronischer Kommunikationsmedien beeinflusst werden.

Insbesondere für Protestbewegungen hat die Bedeutung sozialer Medien als organisatorisches Mittel für Mobilisierungszwecke und Kommunikation in den vergangenen Jahren stetig 
zugenommen (Tremayne 2013; Tufekci 2017; Ebner 2019; Dang-Anh 2019). Aber auch die Polizei bezieht soziale Medien inzwischen immer stärker in ihre Arbeit ein (Ingold 2017; Rüdiger/Bayerl 2018). So auch in Hamburg: Im Rahmen der G20-Proteste wurden soziale Medien von beiden Seiten intensiv genutzt, insbesondere der Nachrichtendienst Twitter. In der sozialwissenschaftlichen Protestforschung wird das Mobilisierungs- und Koordinierungspotenzial sozialer Medien nach wie vor nur unzureichend berücksichtigt, obwohl insbesondere Twitter spätestens seit den Protesten im sogenannten Arabischen Frühling 2011, den globalen OccupyProtesten seit 2011 und den Indignados-Protesten in Spanien, die ebenfalls 2011 begannen, aus Protestkontexten dieser Art kaum noch wegzudenken ist. Dieses Forschungsdefizit hängt auch damit zusammen, dass die Nutzung von Social-Media-Daten eine ganze Reihe von methodischen Problemen mit sich bringt, wie beispielsweise die nur schwer zu schließende Lücke zwischen diskursivem Ereignis einerseits und konkreten SprecherInnenpositionen und Handlungen andererseits. Der folgende Beitrag erhebt vor diesem Hintergrund weder den Anspruch, die Lücke zwischen Diskurs und Praxis methodologisch zu schließen, noch gehen wir implizit von starken Kausalitätsannahmen zwischen Diskurs und Praxis aus. Gleichwohl behaupten wir, einen methodisch wie analytisch innovativen Blick auf die diskursiven Dynamiken in Twitter aufzeigen zu können, die - so unsere These - die Ausweitung der Gewalt während der Protestwoche in Hamburg begünstigt haben. ${ }^{4}$ Wie unsere empirische Analyse der Twitter-Kommunikationsbeziehungen zeigt, entfaltet sich mit fortschreitendem Protestverlauf eine Polarisierung der öffentlichen Debatte, die sich fast nur noch um die Gewaltfrage dreht: Wer ist verantwortlich, wenn es zu Gewalt kommt? Und wie hängt Gewalt, die von Protestierenden ausgeht, mit der Gewalt zusammen, die von der Polizei ausgeübt wird? Mit anderen Worten: Das Knäuel aus Gewalt und Gegengewalt in Hamburg ist überlagert von einer antagonistischen Diskursdynamik, die ihrerseits durch mediale Verstärkereffekte begünstigt wird. Besonders im sozialen Medium Twitter weicht dabei die inhaltliche Diskussion und die Konfrontation mit abweichenden Positionen einer Art kognitiven Schließung, sodass der Twitter-Diskurs nur noch um die Gewaltfrage kreist und dabei zwei digitale Echokammern produziert, in denen sich der jeweilige normative Standpunkt bezüglich der Gewaltfrage radikalisiert. Diese Polarisierung von diametral entgegengesetzten normativen Bewertungen des Geschehens entsteht zunächst einmal parallel zum Protestverlauf, geht dann aber auch über den Zeitraum des G20-Gipfels hinaus und nimmt an Schärfe weiter zu.

Um diese These schrittweise zu entfalten, gehen wir in fünf Schritten vor. In einem ersten Abschnitt werden zentrale Ansätze aus der Protest- und Bewegungsforschung sowie aus der sozialwissenschaftlichen Gewaltforschung skizziert, die das Augenmerk auf die situativen und prozessualen Dynamiken von Gewaltphänomenen legen. Anschließend wird die hier vertretene These begrifflich entfaltet, indem das zentrale Ergebnis der empirischen Analyse vorweggenommen und mit Blick auf die Fragestellung zuspitzend interpretiert wird. In einem dritten Teil wird das methodische Vorgehen genauer beschrieben, bevor die gesamte Argumentation in einem vierten Abschnitt durch eine detaillierte Beschreibung der Ergebnisse der empirischen Analyse plausibilisiert werden soll. Abschließend wird unser Argument noch einmal kurz zusammengefasst und mit einer selbstkritischen Einschränkung versehen.

\footnotetext{
${ }^{4}$ Wir benutzen die Präposition in im Zusammenhang mit Twitter, da wir hervorheben möchten, dass sich die diskursiven Ereignisse und Dynamiken in einem Medium vollziehen.
} 


\section{Theoretischer Ansatz: Perspektiven aus der Protest- und Gewaltforschung}

Das in verschiedenen Phasen der Protestwoche von Hamburg nach und nach entstehende Knäuel aus Gewalt und Gegengewalt stellt ein komplexes dynamisches Geschehen dar, das sich nicht in einfache Kausalketten zerlegen und folglich nur sehr bedingt mit ideologischen Motiven und strategischen Intentionen der Beteiligten erklären lässt. Es scheint deshalb naheliegend, auf analytische Perspektiven zurückzugreifen, die sowohl in der Protest- und Bewegungsforschung als auch in der sozialwissenschaftlichen Gewaltforschung in den vergangenen zwei bis drei Jahrzehnten zunehmend an Bedeutung gewonnen haben und in denen das Augenmerk vor allem auf die situativen und prozessualen Dynamiken des Gewaltgeschehens gelegt wird. Was die Gewaltforschung angeht, so ist zum einen die sogenannte Neuere Gewaltsoziologie gemeint (Koloma Beck 2019), die sich in den 1990er Jahren vor allem in Deutschland entwickelt hat (von Trotha 1997; Nedelmann 1997; Sofsky 1996); zum anderen ist hier an die US-amerikanisch geprägte Mikrosoziologie der Gewalt zu denken, die inzwischen hauptsächlich mit den Arbeiten von Randall Collins (2008) in Verbindung gebracht wird (Hartmann 2019a). ${ }^{5}$ Auf Seiten der Bewegungsforschung wurden die theoretischen und methodologischen Weichen für die hier anvisierte Perspektive ursprünglich von Charles Tilly, Doug McAdam und einigen anderen gelegt, die einst für einen Paradigmenwechsel in der Bewegungs- und Protestforschung sorgten, indem sie ihre Forschung von klassischen Ansätzen kollektiven Handelns weggeführt und stattdessen prozesstheoretische Perspektiven in Stellung gebracht haben (Tilly 1978, 2003; McAdam 1982; McAdam et al. 2001). Aktuell prägen die Arbeiten von Donatella della Porta die Forschungsagenda in diesem Bereich (della Porta 1995, 2008, 2012, 2013).

Die jüngere gewaltsoziologische Forschung legt bevorzugt den Begriff der Situation als zentrale Analysekategorie zugrunde. Sie leitet damit Ende der 1980er bzw. Anfang der 1990er Jahre eine Art situationistische Wende ein, die den Gegenstand Gewalt zunächst als eigenständiges soziales Phänomen in den Mittelpunkt zu rücken versucht. Zu diesem Zweck legt sie ihr Augenmerk auf den physischen bzw. leiblichen Aspekt von Gewalt, statt sich wie die seinerzeit vorherrschenden Ansätze auf die vermeintlichen (z.B. soziostrukturellen) Ursachen hinter der Gewalt zu fokussieren. ${ }^{6}$ In der deutschsprachigen Forschung wird an der von den Protagonisten dieser Wende so bezeichneten Mainstream-Forschung bemängelt, dass diese die Gewalt selbst und ihre besonderen Merkmale, „etwa ihre Anlasslosigkeit und Entgrenzung, aber auch ihre Situationsoffenheit“ (Knöbl 2017: 5), aufgrund ihrer Ursachenfixierung nicht angemessen erfassen könne (von Trotha 1997: 18). Ähnliche Argumente werden fast zeitgleich auch in der internationalen Diskussion vorgebracht, insbesondere von Jack Katz, der Gewalt bereits in den 1980er Jahren aus der Perspektive einer Entgrenzungserfahrung heraus beschrieben hat, die vor allem mit Macht- und Herrschaftsphantasien verbunden ist. Der Suche nach Motiven für Gewalthandeln, die über klassische Befragungsmethoden der Sozialwissenschaften ermittelt wer-

\footnotetext{
${ }^{5}$ Ursprünglich von einer ganzen Reihe unterschiedlicher Autorinnen und Autoren wie Richard Felson und Jack Katz (Felson/Steadman 1983; Katz 1988), Brigitta Nedelmann, Wolfgang Sofsky oder Trutz von Trotha auf den Schild gehoben, stellt Collins' mikrosoziologische Gewalttheorie den zurzeit wohl einflussreichsten Ansatz aus der situationistischen Gewaltforschung dar.

${ }^{6}$ Randall Collins (2008: 23) spricht auch von „background explanations“.
} 
den sollen, erteilt Katz $(1988,2001,2015)$ damit eine klare Absage. Randall Collins (2008) übernimmt später in seiner Mikrosoziologie der Gewalt diesen Zweifel an der Brauchbarkeit von Motiven als Ursachen von Gewalt (Knöbl 2017, 2019).

Inzwischen ist die Betrachtung von Gewalt als unmittelbare leibliche Interaktion mit situationsspezifischen Eigenschaften zum vorherrschenden Verständnis in der internationalen Gewaltdiskussion avanciert. Und selbst die Theoriedebatte innerhalb der sozialwissenschaftlich orientierten Gewaltforschung ist seit nunmehr zwei Jahrzehnten vom situationistischen Paradigma geprägt, während Bezugnahmen auf übergreifende gesellschaftliche Kontexte immer weiter in den Hintergrund gedrängt wurden (Knöbl 2019; Schützeichel 2019).

Die Gewaltforschung hat mittlerweile einen theoretischen Stand erreicht, auf dem es nicht mehr plausibel ist, analytische Aussagen über Gewalthandlungen zu machen, ohne näher auf die spezifischen Merkmale der jeweiligen Situation einzugehen, in der sie sich ereignen. (Hoebel 2019: 99)

Zugleich aber läuft der Trend zugunsten des situationistischen Ansatzes Gefahr, einer reduktionistischen Theoriebildung Vorschub zu leisten (Sutterlüty 2017; Hartmann 2019a). In jüngster Zeit mehren sich deshalb die Stimmen, die Skepsis am Erklärungspotenzial des situationistischen Paradigmas anmelden und für methodologische Erweiterungen der damit eröffneten Forschungsperspektive plädieren (Hoebel/Malthaner 2019).

Diesen aktuellen Diskussionsstrang innerhalb der Gewaltforschung wollen wir gezielt aufgreifen, indem wir einerseits an der Prämisse festhalten, dass sich gewaltspezifische Handlungsdynamiken wie das hier behandelte G20-Protestgeschehen in Hamburg nur über den analytischen Zugriff auf die Situation angemessen begreifen lassen, in der diese sich entfalten; andererseits gehen wir im Anschluss an Rainer Schützeichel (2019) davon aus, dass Situationsdefinitionen sozialer Akteure notwendigerweise sozial situiert sind und gerade in dieser Eigenschaft zum Bestandteil jener Situationen werden, die von situationistischen Analysen als Untersuchungseinheiten definiert werden (und zwar in der Regel von einem externen Beobachtungsstandpunkt aus). Daraus ergibt sich methodologisch die Notwendigkeit, die Situationsdefinitionen der an gewaltförmigen Interaktionen Beteiligten in die Analyse mit einzubeziehen - und zwar nicht nur von direkt in das Geschehen beteiligten Akteuren (im Sinne von Tätern und Opfern), sondern insbesondere auch von Dritten, die das Gewaltgeschehen beobachten und normativ bewerten. Mit Blick auf die von uns verfolgte Fragestellung, die auf den spezifischen Beitrag der Twitter-Kommunikation zur Eskalation des Protestgeschehens gerichtet ist, stellt sich die Frage, wie sich das Ausüben, Erleiden und Beobachten bzw. Benennen von Gewalt gleichermaßen situativ (also im Geschehen vor Ort) und die Situation transzendierend (also die Situation vor Ort sowohl zeitlich als auch örtlich überschreitend) vollzieht. In dieser Perspektive realisiert sich das Geschehen vor Ort kontextgebunden, wobei normative Bewertungen des Geschehens und Erwartungen an zukünftiges Geschehen zentrale Situationselemente bilden, die das Geschehen beeinflussen. In diesem Zusammenhang spielt der sogenannte engagierte oder „interessierte Dritte“, wie Jan Philipp Reemtsma (2008: 471) den beobachtenden Dritten unter anderem nennt, eine zentrale Rolle, da dessen normative Bewertung des Geschehens mit darüber entscheidet, welche Handlungsspielräume sich in gewaltförmigen Situationen ergeben, ob sich der Spielraum für Gewalthandeln mit anderen Worten erweitert oder verringert: 
[...] the observer' might become decisive for the evolution of a violent interaction. His or her relative position to the, performer' and the , target' is crucial in defining the potential and limitations of a violent situation: an ,observer' sympathizing with the ,target' potentially limits the option of the ,performer', whereas a timid or even applauding,observer' affirms and encourages the violent assault. (Koloma Beck 2011: 351)

Beim zweiten Forschungsstrang, der hier aufgegriffen werden soll, handelt es sich um die im erweiterten Sinne soziologisch orientierte Bewegungs- und Protestforschung, die in Verbindung mit der neueren Terrorismusforschung innovative Wege zu einer Soziologie politischer Gewalt aufzeigt. Ihr Augenmerk gilt dabei in erster Linie der Prozessualität gewaltsamer Interaktionen (della Porta 1995, 2012, 2013; Bosi/della Porta 2012; Bosi et al. 2014). Die Arbeiten aus diesem Bereich zielen darauf ab, konkrete Formen kollektiver Gewalt wie Genozide, Revolutionen, gewalttätige Demonstrationen, soziale Unruhen oder terroristische Gewalt primär aus einer prozesstheoretisch orientierten Perspektive heraus zu begreifen. Eine solche Perspektive ist zunächst unweigerlich mit dem Namen Charles Tilly verbunden. Bevor dieser in den 1960er Jahren damit begann, historische Gewaltereignisse wie die Französische Revolution (Tilly 1964) zu erforschen, wurde kollektive Gewalt in aller Regel als eine Art Pathologie des Sozialen behandelt, und zwar meist im Rahmen sogenannter Strain- und Breakdown-Theorien kollektiven Handelns. Diese fokussierten ausschließlich auf makrostrukturelle Veränderungen, die angeblich zu einer Schwächung sozialer Bindungen und damit einhergehender normierender Handlungszwänge führen, wodurch - so die zentrale Annahme - Entfremdung und soziale Haltlosigkeit zunehmen und die Menschen verstärkt zu normverletzenden Verhaltensweisen neigen (Smelser 1959; Gurr 1970; Heitmeyer 1996, 1997). Tilly geht hingegen davon aus, dass Formen kollektiver Gewalt zum gesellschaftlichen Repertoire von Machtkämpfen gehören (Sewell 1990: 528). Er macht damit deutlich, dass die gewaltinteressierte Protest- und Bewegungsforschung es mit ganz grundlegenden Prozessen sozialen Wandels zu tun hat. In Anlehnung an Tilly entsteht daraufhin gegen Ende der 1970er Jahre der so genannte resource mobilization approachein Strang innerhalb der Bewegungsforschung, der vor allem die rationalen Handlungsorientierungen kollektiver Akteure betont, auch wenn das Thema Gewalt in diesem Kontext zunächst so gut wie keine Rolle spielt (McCarthy/Zald 1977; Tilly 1978; McAdam et al. 1996; Buechler 2004).

Im Geiste dieser Forschungslinie kommt es spätestens in den 1980er Jahren zu einer dezidiert prozesstheoretischen Wende innerhalb der Bewegungsforschung, die sich immer häufiger auch mit terroristischen Gruppierungen, mit deren militanten Strategien und der besonderen Dynamik der gewaltsamen Eskalation von Protestverläufen beschäftigt (Neidhardt 1981; della Porta/Tarrow 1986; della Porta 1995). Dabei übernimmt erstmals der Begriff der Eigendynamik eine tragende Rolle für die Analyse anhaltender Prozesse gewaltsamer Interaktionen. Eigendynamische soziale Prozesse entstehen zugespitzt formuliert dadurch, dass sie „Folgen erzeugen, die zum Bestandteil ihrer eigenen Verursachungsstruktur werden“ (Mayntz 1987: 660). Besonders Friedhelm Neidhardt plädiert in diesem Sinne dafür, das Augenmerk weg von individuellen und soziostrukturellen Aspekten und stattdessen auf Prozessabläufe zu richten, um die Emergenz gewaltsamer (in seinem Fall terroristischer) Auseinandersetzungen stärker in den Mittelpunkt rücken zu können: 
Terrorismus entsteht mit einem durchschnittlichen Sortiment von Leuten unter den unterschiedlichsten gesellschaftlichen Ausgangsbedingungen. Das Entscheidende liegt offensichtlich nicht in individuellen und soziostrukturellen Prädispositionen, sondern in Prozeßabläufen, in denen zahlreiche Bedingungen ein Handlungssystem begründen und in, zirkulären Interaktionen' aufeinander und auf sich selber einwirken. Sie sind Ursache und Wirkung zugleich, und zwar in Abhängigkeit von den wechselnden Konstellationen, mit denen sie aufeinandertreffen. (Neidhardt 1981: 244)

Innerhalb der etwas jüngeren Tradition der Bewegungsforschung wurden prozesstheoretische Perspektiven insbesondere im Anschluss an das von Charles Tilly und Sidney Tarrow entwickelte und international breit rezipierte Paradigma der contentious politics aufgegriffen und kontinuierlich weiterentwickelt (McAdam et al. 2001; Tilly/Tarrow 2006; Bosi et al. 2014). Tilly und Tarrow verstehen unter contentious politics ,,interactions in which actors make claims bearing on someone else's interests, leading to coordinated efforts on behalf of shared interests or programs, in which governments are involved as targets, initiators of claims, or third parties“ (Tilly/Tarrow 2006: 4). Zur begrifflichen Systematisierung solcher coordinated efforts schlagen die Autoren das Konzept der contentious repertoires vor, das für ein verfügbares Set an Protestformen steht, auf das Akteure eines spezifischen Bewegungszusammenhangs zurückgreifen können (Tilly/Tarrow 2006: 11). Gewalt ist aus dieser Perspektive weder als eine Wesenseigenschaft bestimmter Individuen oder Protestgruppen zu verstehen noch als Folge eines rein strategischen Handlungskalküls, sondern als Eskalation von Handlungsrepertoires unter bestimmten und in der Regel prozessbedingten Umständen. Genau das meint „violence as escalation of action repertoires“ (della Porta 2008: 222), wie della Porta es später ausdrücken wird:

Seeing violence as an escalation of the protest repertoire points to the fluid borders between strategies, and the reciprocal adaptation and learning as a result of interaction between social movements and external actors, namely the police and other adversaries. (della Porta 2012: 246)

Della Porta vertritt eine dezidiert bewegungstheoretisch orientierte Perspektive auf politische Gewalt und schlug bereits in den 1990er Jahren vor, den Begriff der „Radikalisierung“ prozesstheoretisch zu konzipieren, um ihn für die Analyse politischer Gewalt fruchtbar zu machen (della Porta 1995: 111). Radikalisierung begreift sie als einen sich selbst verstärkenden Prozess der Interaktion zwischen gewalttätigen Gruppen und deren gesellschaftlicher Umgebung oder zwischen verschiedenen verfeindeten Akteuren - z.B. zwischen staatlichen und nicht-staatlichen Gruppierungen oder zwischen politischen Gegnern (della Porta/LaFree 2012: 7). Am Beispiel der linksterroristischen Rote Armee Fraktion zeigt sie, dass äußere Bedingungen wie Mobilisierungsschwäche, Ressourcenknappheit und die soziale Isolierung der Gewaltgruppe „triggered organizational processes that in turn favored the diffusion of violence " (della Porta 1995: 83). Sie betont, dass diese Prozesse in aller Regel auch die Erwartungs-, Wahrnehmungs- und Deutungsschemata transformieren, die der Konstruktion von politischer Gegnerschaft zugrunde liegen (della Porta 1995: 136). Eine solche kognitive Schließung ist dabei primär als Resultat von sozialer Schließung zu begreifen, wodurch der auch für die von uns vertretene These 
entscheidende Zusammenhang zwischen Kognition und sozialer Interaktion in den Fokus rückt:

This communal solidarity also effected the militants' cognitive closure insofar as all the information the militant activists received was filtered through the group and this information defined their external reality, thus providing shared frames of meaning. Although radical beliefs are not themselves the cause of violence, the ideological frames through which the militants interpreted their daily encounters with political violence tended to dramatize the significance of these events. (della Porta 2012: 254)

Die in diesem Beitrag vorgelegte Analyse zielt darauf ab, genau diesen Prozess einer kognitiven Schließung für den hier behandelten Fall der gewaltsamen Ausschreitungen im Kontext der G20-Proteste in Hamburg sichtbar zu machen und anhand einer empirischen Analyse der Kommunikationsbeziehungen im sozialen Medium Twitter zu rekonstruieren. Die von uns durchgeführte Analyse der netzwerkartigen Kommunikationsbeziehungen, die sich im Zuge der Gipfelproteste im sozialen Medium Twitter bilden, untermauert die sowohl in der Protestund Bewegungsforschung als auch in der Gewaltforschung inzwischen gängige Annahme, dass eskalierende Konfliktverläufe notwendig mit Transformationen kognitiver Wahrnehmungsund Deutungsmuster einhergehen, die durch ihren polarisierenden Effekt zu einem zentralen Bestandteil der gesamten Verursachungsstruktur gewaltsamer Konflikte werden. Im Mittelpunkt steht hierbei die These, dass sich parallel zur Eskalation des Protestgeschehens auf den Straßen Hamburgs eine antagonistische Diskursdynamik im sozialen Medium Twitter ereignet, die durch die Bildung zweier feindseliger Lager gekennzeichnet ist. Diese Polarisierung jener Diskursarena, die wir mit Hilfe umfassender digitaler Verlaufsdaten der Twitter-Kommunikation während der Protestwoche untersucht haben, begünstigt sich selbst verstärkende Spiralen aus Solidarisierung und Feindbildkonstruktion, die insgesamt charakteristisch für die Eskalationsphasen der Protestwoche sind. Diese Spirale, die spätestens am 7. Juli im Zusammenhang mit den abendlichen Ausschreitungen im Schanzenviertel (Malthaner 2019) zur Entfaltung kommt, entzündet sich in erster Linie an der Gewaltfrage. Deren zusätzlich polarisierte Auslegung durch den Twitter-Diskurs erzeugt eine Art normativen Gruppenstandpunkt, auf den sich Akteure beziehen können, die vor Ort tatsächlich Gewalt ausüben. Für sie bildet die durch öffentliche Kommunikationen dieser Art erzeugte Sinngebung eine wesentliche symbolische Ressource, um den Spielraum für das eigene (Gewalt-)Handeln situativ zu erfassen.

\section{These: die Ausweitung der Zone gebotener oder wenigstens erlaubter Gewalt im sozialen Me- dium Twitter}

Aus einer primär situations- und prozessorientierten Perspektive sind Umwelteinflüsse sowie individuelle Handlungsdispositionen und -motive zwar nicht unbedeutend für die Entstehung und den Verlauf gewaltsamer Interaktionen. Entscheidend ist aber, dass diese Prozesse oft von sozialen Dynamiken getragen werden, die sie primär selbst erzeugen. Dabei können sich die Ausgangsbedingungen eines Konflikts oder eines Protestgeschehens maßgeblich verändern, etwa weil Akteurskonstellationen sich verschieben oder sekundäre Motive entstehen, welche 
die originären Zielsetzungen der am Konflikt beteiligten Akteure überlagern oder sogar verdrängen können. Das in anhaltenden Prozessen gewaltsamer Konflikte entstehende Knäuel aus Gewalt und Gegengewalt, wie wir es in Hamburg beobachten konnten, wird dann im Sinne einer spezifischen Prozessdynamik begreifbar. So wird das ansteigende Gewaltniveau in den erbitterten Auseinandersetzungen zwischen politischen Gegnern oder zwischen Protestbewegungen und staatlichen Sicherheitsbehörden nicht auf Motive oder strategische Intentionen von Akteuren zurückgeführt, sondern lässt sich als das Resultat von eigendynamischen Prozessen gewaltsamer Eskalation deuten:

Eskalationen sind Prozesse zirkulärer Interaktionen, bei denen sich alle Beteiligten in Richtung wachsender Abweichung stimulieren. Jeder Schritt der einen Seite erfährt positiven feed-back durch die andere. Es ereignen sich Reiz-Reaktions-Sequenzen, mit denen der Konflikt in sich verstärkende Turbulenzen trudelt. (Neidhardt 1981: 245)

Soziale Prozesse gewaltsamer Interaktionen entfalten sich aus dieser Perspektive nicht etwa linear, sondern sind durch Brüche, Unterbrechungen und „unvorhersehbare Interferenzen“ charakterisiert (Schützeichel/Jordan 2015: 11). Es handelt sich deshalb um hochkontingente emergente Phänomene, die nur über die detaillierte Rekonstruktion der jeweiligen Fallstruktur zu erfassen sind. ${ }^{7}$ Demnach haben Gewaltanalysen erstens bei einer systematischen Rekonstruktion der Fallstruktur (casing) und einer detaillierten Beschreibung jener Ereignisse anzusetzen, die die Emergenz von Gewalt beeinflussen. Diese Ereignisse müssen zweitens aus einem spezifischen Interaktionszusammenhang heraus begriffen werden. Die Gewaltakteure dürfen mit anderen Worten nicht isoliert betrachtet werden, sondern stets „within a broader relational field of actors“ (Malthaner 2017: 375). Aus diesem Interaktionszusammenhang heraus müssen drittens die Prozesse der kognitiven Transformation betrachtet werden, die auch Stefan Malthaner jüngst mit Blick auf die aktuelle Forschungsliteratur zu Radikalisierungsprozessen besonders hervorhebt:

Patterns of interaction with opponents and rivals are seen as producing - and as being reinforced by - shifts in perception and beliefs. In fact, processes of radicalization are understood to result from the interaction of environmental, cognitive, and relational mechanisms [...], although priority is certainly given to the way in which relational dynamics shape and put into effect cognitive mechanisms. (Malthaner 2017: 375)

Die hier treffend zugespitzte Auffassung von Radikalisierung als ein sich in konkreten Interaktionszusammenhängen entfaltender Prozess, beim dem äußere Einflüsse im Sinne von environtmental mechanisms mit relationalen und kognitiven Mechanismen zusammenwirken,

\footnotetext{
${ }^{7}$ Dies war das Kernanliegen des Projekts Mapping \#NoG20, dem ein dreigliedriges modulares Untersuchungsdesign zugrunde liegt. Das Modul Ausgangskonstellation widmet sich den im Vorfeld des Gipfels prägenden Debatten sowohl innerhalb des Protestspektrums als auch auf Seiten der staatlichen (Sicherheits-)Behörden. Die Module Situationsanalysen und Mediale Deutungen widmen sich den Interaktionen und Deutungen in der Woche vom 2.-9. Juli, wobei zwischen einzelnen Protestereignissen und ihrem konkreten Verlauf einerseits und den medialen Deutungen in Presse und sozialen Medien andererseits unterschieden wird.
} 
bildet den analytischen Kern all jener Arbeiten, die Gewalt in Fortführung der Tilly'schen Programmatik an der Schnittstelle zwischen Bewegungs- und soziologischer Gewaltforschung untersuchen.

Dieser Forschungsmethodologie ist auch die hier bemühte Perspektive insofern verpflichtet, als sie das Augenmerk auf den wechselseitigen Zusammenhang aus relationalen und kognitiven Mechanismen der Eskalation des Hamburger Protestgeschehens legt. Dabei liegt die empirische Besonderheit in der vergleichsweise kurzen Zeitspanne, in der sich die kognitive Transformation der Wahrnehmungs-, Deutungs- und Erwartungsschemata im Zusammenhang mit einer strukturellen Polarisierung der Interaktionsbeziehungen vollzieht. Der Kurznachrichtendienst Twitter übernimmt dabei eine Schlüsselrolle, da dieser Nachrichten quasi in Echtzeit verbreitet und so eine ungefilterte öffentliche Kommunikation über aktuelle Ereignisse ermöglicht. Indem einzelne Nachrichten (Tweets) innerhalb eines Twitter-Netzwerks verbreitet, kommentiert oder als sogenannte Re-Tweets geteilt werden und somit verschiedene Diskursarenen miteinander verknüpfen, entstehen - parallel zum Protestverlauf - netzwerkartige Kommunikationsbeziehungen, die sowohl auf Seiten der Öffentlichkeit als auch auf Seiten der Akteure vor Ort die Wahrnehmung und Bewertung des Geschehens beeinflussen. Einzelne Tweets können dabei eine erhebliche Strahlkraft entfalten, und zwar relativ unabhängig von ihrem Wahrheitsgehalt. Verstärkt wird dieser Effekt durch einflussreiche Medienakteure, deren Twitter-Aktivitäten durch überdurchschnittlich große Follower-Zahlen ein sehr breites Publikum erreichen. Vor allem polizeiliche Falschnachrichten landen so häufig als Fakten in den Medien (nicht zuletzt auch in den etablierten Printmedien), während anschließende Richtigstellungen im beschleunigten Medienstrudel meist untergehen. ${ }^{8}$

In ihrer Doppelfunktion als Informationsquelle und Kommunikationsplattform dienen soziale Medien wie Twitter in Protestzusammenhängen einerseits sowohl den Protestierenden als auch der Polizei als eine wesentliche Grundlage für Deutungsprozesse, die der Protest- bzw. Polizeipraxis zugrunde liegen; andererseits bilden sie zentrale Diskursarenen, in denen sich symbolische Kämpfe um Deutungshoheit entfalten. Das, was als Grundlage für die Interpretation des Geschehens und der Legitimation eigener Handlungen dient, ist also zugleich immer auch Gegenstand von Interpretationskämpfen, die nicht zuletzt über die sozialen Medien selbst ausgefochten werden. Der dazugehörige Diskurs speist sich vor allem aus normativ aufgeladenen Beschreibungen der Geschehnisse, die von Beobachtenden oder auch von direkt in das Protestgeschehen involvierten Akteuren über soziale Medien kommuniziert werden. Die spezifische Diskursarena Twitter ist also weder als reines Abbild der diskursiven Prozesse vor Ort noch als autonome Diskursarena ohne jeglichen Bezug zum realen Geschehen zu begreifen. Vielmehr müssen wir davon ausgehen, dass die verschiedenen Diskursebenen miteinander verschränkt sind und sich wechselseitig beeinflussen. Denn gerade im Fall von Twitter lassen sich Interaktionen vor Ort und ihre mediale Aufarbeitung zeitlich kaum noch voneinander trennen. Beobachtungen und normative Bewertungen, die beispielsweise zunächst von mehr oder weniger in das Geschehen involvierten Akteuren ausgehen, werden als Tweet Bestandteil eines in Echtzeit geführten Diskurses, in dem um die legitime Auslegung der Geschehnisse gerungen

\footnotetext{
${ }^{8}$ Zum Problem, dass Falschnachrichten sich über Twitter häufig schneller und wirksamer verbreiten als wahrheitsgemäße, siehe Vosoughi et al. (2018). Für Beispiele polizeilicher Fake-News auf Twitter im Kontext der Gipfelproteste in Hamburg, siehe auch Reuter (2018).
} 
wird. Diese wiederum erhalten ihre soziale Bedeutung immer erst im Kontext von Diskursen, die über die direkte Kommunikation unter den Interaktionsteilnehmenden hinausreichen. Die Besonderheit von Twitter ist vor diesem Hintergrund darin zu sehen, dass diese Diskurse über digitale Netzwerke umgehend zu den Akteuren vor Ort zurückfließen und dadurch unweigerlich auf deren Deutungen, Handlungsorientierungen und Interaktionen einwirken.

Im Zentrum der im Medium Twitter geführten Diskurse während der Hamburger Protestwoche steht ohne Zweifel die Gewaltfrage. Diese dreht sich im Kern um die Frage, wie mögliche Gewalt, die von Protestierenden ausgeht, mit Gewalt zusammenhängt, die möglicherweise von der Polizei ausgeht. Hierbei ist stets umstritten, was legitime und was illegitime Gewalt ist, wer sie ausgeübt und vor allem: wer ursprünglich mit der Gewalt begonnen hat. Dabei ist der normative soziale Standpunkt, von dem aus derartige Sinnzuschreibungen vorgenommen werden, in aller Regel ein Gruppenstandpunkt, der über diskursive Prozesse erzeugt wird und der für jene Akteure, die vor Ort tatsächlich Gewalt ausüben, die notwendigen legitimierenden Handlungsressourcen generiert. Dieser Standpunkt ist mit anderen Worten an die Repräsentation der sozialen Wirklichkeit von Gruppen gebunden, die ihrerseits das Produkt von sozialer Interaktion ist (Hartmann 2016, 2017, 2019b). Jan Philipp Reemtsma schreibt an einer entscheidenden Stelle seiner Überlegungen:

Das Wichtigste an der Erzeugung, Weckung oder Stimulierung von Gewaltbereitschaft ist die Versicherung, man befinde sich (möglicherweise anders, als der erste Augenschein lehrt) in einer Zone gebotener oder wenigstens erlaubter Gewalt. (Reemtsma 2006: 19; vgl. auch Reemtsma 2008: 195, 457)

Genau diese Versicherung, man befinde sich in einer Zone gebotener oder wenigstens erlaubter Gewalt, entspricht einem hermeneutischen Verhältnis zwischen Akteur und Handlungsumwelt, das aus einem konkreten Zusammenhang von sozialen Interaktionen und Repräsentationen hervorgeht. Anders formuliert: Die Zonen gebotener oder wenigstens erlaubter Gewalt entstehen, weil es kollektive Vorstellungen gibt, die Gewalt als gebotenes oder wenigstens erlaubtes Mittel sozialen Handelns definieren, und weil es soziale Beziehungen und damit verknüpfte Kommunikationen/Interaktionen gibt, die derartige normative Zuschreibungen symbolisch vermitteln. Für das hier vorgetragene Argument ist dabei die Möglichkeit entscheidend, dass dieses hermeneutische Verhältnis zwischen Akteur und Handlungsumwelt nicht zuletzt über soziale Medien erzeugt wird, und zwar insbesondere in Protestkontexten (Ebner 2019; DangAnh 2019). Den Spielraum für (aus Akteursperspektive) erlaubtes oder gar gebotenes Gewalthandeln zu erfassen, ist auch an eine über soziale Medien vermittelte Weltwahrnehmung gebunden - an einen situierten sozialen Standpunkt, der zugleich ein Gruppenstandpunkt ist und über den die anhaltend zu verhandelnde Gewaltfrage normativ vermittelt wird. Anders ausgedrückt: Der digitale Diskurs im Medium Twitter spielt bei der Vermittlung zweier antagonistisch aufeinander bezogener Standpunkte, von denen aus die Gewaltfrage jeweils normativ bewertet wird, eine zentrale Rolle. 


\section{Methode: trianguliertes Verfahren der Netzwerk- und Inhaltsanalyse des Twitter-Diskurses}

Die Datengrundlage für die Analyse der Twitter-Kommunikation während der Gipfelproteste bilden über 700.000 Tweets aus dem Untersuchungszeitraum 28.6. bis 13.7.2017, die mit einer sogenannten Twitter-Streaming-API erhoben wurden. APIs (Application Programming Interfaces, zu deutsch Anwendungsprogrammierschnittstellen) dienen im Wesentlichen dem Austausch von Daten und Inhalten zwischen Softwarekomponenten und/oder Websites. Damit stellen sie einen wichtigen Teil der Infrastruktur von Webplattformen wie Twitter dar, beispielsweise indem sie die Übermittlung von Nachrichten und Tweets organisieren. API-Zugänge ermöglichen den Programmierzugriff auf entsprechende Abläufe und erlauben es, für normale NutzerInnen verschlossene Datenpools einzusehen. Twitter bietet verschiedene APIZugänge mit jeweils unterschiedlichen Zugriffsrechten an. Bei der Twitter-Streaming-API handelt es sich um einen von Twitter zur Verfügung gestellten Zugang, der die Speicherung von Twitter-Nachrichten (Tweets) in Echtzeit nach zu definierenden Parametern ermöglicht. Übersteigt die dadurch erfasste Auswahl an Twitter-Nachrichten einen von Twitter bestimmten Grenzwert, wird eine automatische Zufallsauswahl generiert (vgl. Morstatter et al. 2013, 2014). Für die Analyse wurde nach deutschsprachigen Tweets aus dem Untersuchungszeitraum 28.06.2017 bis 13.07.2017 gefiltert, die den Begriff G20 (bzw. g20) enthalten. Auf diese Weise konnte ein Datensatz mit über 700.000 Tweets erstellt werden. ${ }^{9}$

Anschließend wurden die erhobenen Daten fünf Episoden zugeordnet, die charakteristischen Phasen des zeitlichen Ablaufs des Protestgeschehens entsprechen. Die Auswahl der Zeitabschnitte basiert dabei auf der Identifikation von Ereignissen, denen eine besondere Relevanz für den Protestverlauf zukommt. Die Aufteilung der Episoden gestaltet sich wie folgt: Episode 1 (Prolog) erstreckt sich vom 28.06. (00:00 Uhr) bis zum 01.07. (24:00 Uhr) und beschreibt den Auftakt der Protestereignisse. Episode 2 (Solidarisierung) umfasst den Zeitraum vom 02.07. (00:00 Uhr) bis zum 05.07. (24:00 Uhr), der insbesondere durch die juristisch umstrittene Räumung des Protestcamps im Elbpark Entenwerder durch die Polizei am Abend des 2. Juli sowie die daran anknüpfende Auseinandersetzung um die Errichtung von Camps an anderen Orten in Hamburg gekennzeichnet ist. Episode 3 (Eskalation) beschreibt die Ereignisse zwischen dem 07.07. (00:00 Uhr) und dem 08.07. (03:00 Uhr), worunter das In-Brand-setzen von Autos durch eine schwarz gekleidete Gruppe von Protestierenden am Morgen des 7. Juli sowie die sich bis in die Nacht hineinziehenden Ausschreitungen und Plünderungen im Schanzenviertel mit anschließendem Einsatz des Polizei-Spezialeinsatzkommandos SEK als besonders relevant identifiziert wurden. Episode 4 (Schock) umfasst den Zeitraum vom 08.07. (03:00 Uhr) bis zum 09.07. (24:00 Uhr), der durch die Veranstaltung der Großdemonstration Grenzenlose Solidarität statt G20 am 8. Juli sowie durch weitere Zusammenstöße im Schanzenviertel geprägt ist. Episode 5 (Epilog) beschreibt schließlich den Nachgang der Protestwoche vom 10.07. (00:00 Uhr) bis zum 13.07. (24:00 Uhr). ${ }^{10}$

\footnotetext{
${ }^{9}$ Ein ausdrücklicher Dank gebührt an dieser Stelle Sebastian Haunss, der die Rohdaten im Rahmen des Projekts Mapping \#NoG20 erhoben und uns zur Auswertung zur Verfügung gestellt hat.

${ }^{10}$ Das Fehlen von Daten für den 06.07.2017, der insbesondere durch die gewaltsame Auflösung der Demonstration „Welcome to Hell“ durch die Polizei gekennzeichnet ist, ist die Folge eines technisch bedingten Ausfalls der Streaming-API.
} 
Für jede Episode wurden jeweils Verfahren der Netzwerkanalyse, eine Auszählung von im Textkorpus enthaltenen Schlüsselwörtern als explorative Methode der quantitativen Textanalyse sowie eine qualitativ-inhaltliche Auswertung systematisch ausgewählter Tweets durchgeführt. Dazu wurden die Analyseprogramme $R$ (R Core Team 2017) mit den tidyverse-Paketen (Wickham et al. 2019) und Gephi (Bastian et al. 2009) verwendet. Durch die spezifische Methodentriangulation aus Netzwerkanalyse, quantitativer Textanalyse und fokussierter inhaltlicher Auswertung können zum einen Prozesse der strukturellen Formierung der Diskursarena Twitter im Verlauf der Gipfelproteste untersucht werden; zum anderen werden durch diese Vorgehensweise semantische Veränderungen in der diskursiven Deutung des Protestgeschehens erkennbar, die mit den strukturellen Merkmalen der Netzwerke direkt in Zusammenhang gebracht werden können. Die Analyseschritte werden im Folgenden näher beschrieben.

Für jede Episode wurden zunächst Netzwerkmodelle auf Grundlage von sogenannten ReTweets, also der Praxis des Weiterleitens von Tweets anderer NutzerInnen erstellt, wobei die Knoten des Netzwerks die (re-)tweetenden NutzerInnen charakterisieren; die Kanten stellen die Verbindungen zwischen ebendiesen dar. Dabei sind die Kanten von den NutzerInnen, die Tweets anderer NutzerInnen re-tweeten, auf die OriginalverfasserInnen des geteilten Tweets gerichtet. Dementsprechend handelt es sich im Fall der vorliegenden Untersuchung um sogenannte gerichtete Netzwerke. Sie bilden die Struktur des Twitter-Diskurses zum Thema G20 sowie die in diesem enthaltenen Kommunikationswege ab. Um die je nach Followerzahl der NutzerInnen variierende Reichweite der (Re-)Tweets sowie wiederholtes Weiterleiten der Tweets von NutzerInnen durch andere NutzerInnen mit berücksichtigen zu können, wurden die Kanten entsprechend des Produkts aus der Followerzahl der re-tweetenden NutzerInnen und der Anzahl von Verbindungen zwischen diesem und den OriginalverfasserInnen des Tweets gewichtet. ${ }^{11}$ Dem Gewichtungsverfahren liegt die Annahme zugrunde, dass Tweets, die von NutzerInnen mit einer hohen Anzahl an Followern geteilt werden, eine hohe Öffentlichkeit generieren und entsprechend gewichtiger sind als Tweets, die von NutzerInnen mit geringer Followerzahl geteilt werden. Die Einbeziehung der Anzahl von Verbindungen zwischen den NutzerInnen in die Berechnung des Gewichtungsfaktors dient darüber hinaus der Sichtbarmachung bestimmter, stark frequentierter Kommunikationswege innerhalb der Diskurs-Arena Twitter. Um unverhältnismäßig hohe Gewichtungen bei NutzerInnen mit sehr hohen Followerzahlen bzw. einer hohen Anzahl von Verbindungen zu anderen NutzerInnen und daraus resultierende Verzerrungseffekte bei der Berechnung der Netzwerkmodelle zu vermeiden, wurde der Gewichtungsfaktor entlang seiner Quintile (Fünftelwerte) kategorisiert, womit er die Form einer ordinalskalierten Variable mit den Werten 1 bis 5 annimmt.

Auf Basis der so generierten Daten wurde in einem weiteren Schritt jeweils ein Netzwerkmodell pro Episode erstellt (siehe Abbildungen 1-5 im Appendix). Um die Darstellbarkeit der teils sehr großen Netzwerke zu gewährleisten und ein Überschreiten der vorhandenen Rechenkapazität zu vermeiden, wurde die Anzahl der abgebildeten Knoten jeweils auf einen Anteil von 2,5\% der Gesamtknotenzahl reduziert. Dazu wurden Knoten mit einer geringen Anzahl an Ver-

\footnotetext{
${ }^{11}$ Als Follower werden NutzerInnen bezeichnet, die anderen NutzerInnen gezielt folgen, um deren (Re-)Tweets unmittelbar auf ihrem Twitter-Profil angezeigt zu bekommen. Die Anzahl an Verbindungen zwischen zwei NutzerInnen erhöht sich, wenn ein/eine NutzerIn innerhalb einer Episode mehrere Tweets eines/einer anderen NutzerIn weiterleitet.
} 
bindungen mit anderen Knoten (dem sogenannten Grad) schrittweise aus den Modellen entfernt, bis dieser Schwellenwert erreicht wurde. ${ }^{12}$ Anschließend wurden die Netzwerke unter Verwendung des Algorithmus Force Atlas 2 (Jacomy et al. 2014) modelliert. Um innerhalb der Twitter-Netzwerke NutzerInnen zu identifizieren, denen als Diskurs-ProduzentInnen dadurch eine besondere Relevanz zukommt, dass ihre Tweets besonders häufig und/oder von NutzerInnen mit besonders hohen Followerzahlen geteilt werden, wurden die Knoten in einem darauffolgenden Schritt entlang ihres gewichteten Eingangsgrads, das heißt entlang der Anzahl an Kanten, die auf die jeweiligen Knoten gerichtet sind, bei gleichzeitiger Berücksichtigung ihrer Gewichtung hierarchisiert (siehe Tabellen 1-5 im Appendix). In den Abbildungen sind Knoten mit einem hohen gewichteten Eingangsgrad mit entsprechend größeren Punkten abgebildet, als jene mit geringeren Werten. Dadurch, dass die Nachrichten dieser NutzerInnen per ReTweet besonders häufig verbreitet werden und dass sie außerdem eine große Anzahl an Followern erreichen können, tragen sie überproportional zur Etablierung spezifischer Beschreibungen und Bewertungen bei.

In einem letzten Schritt der Netzwerkanalyse wurde schließlich die modulare Struktur der Netzwerke berechnet, wobei ein von Blondel et al. (2008) entwickelter Algorithmus verwendet und mit variierenden Auflösungen gearbeitet wurde (Lambiotte et al. 2009). Auf diese Weise konnten spezifische Gemeinschaften (communities) innerhalb der Netzwerke identifiziert werden, die sich durch die ungefilterte Verbreitung von Beschreibungen und/oder normativen Bewertungen von Ereignissen formieren und durch ein Mindestmaß an innerer Geschlossenheit gekennzeichnet sind. Sie sollen im Folgenden als Teilnetzwerke bezeichnet werden. Um Unterschiede in der Struktur der episodenspezifischen Netzwerke sowie Veränderungen in der Relation der Teilnetzwerke zueinander zu beschreiben, wurde abschließend die Dichte des Gesamtnetzwerks sowie die Dichte der Verbindungen zwischen den Teilnetzwerken berechnet. Da es sich im Fall der untersuchten Netzwerkmodelle um gerichtete Netzwerke handelt, wird die Relation zweier Teilnetzwerke zueinander durch zwei Dichtewerte beschrieben, die die jeweilige Richtung der Verbindungen kennzeichnen. Zur Berechnung der Dichte des Gesamtnetzwerks bzw. der Verbindungen zwischen zwei Teilnetzwerken wird die mögliche Anzahl an Verbindungen zwischen allen Knoten des Gesamtnetzwerks bzw. zwischen den Knoten der beiden Teilnetzwerke ins Verhältnis zu der Anzahl an tatsächlich bestehen Verbindungen gesetzt. Dementsprechend kann die Dichte Werte zwischen 0 und 1 annehmen, wobei der Wert 0 ein aus isolierten Knoten/Teilnetzwerken bestehendes Netzwerk ohne jegliche Verbindungen zueinander beschreibt und der Wert 1 ein Netzwerk, in dem alle Knoten des Gesamt-/Teilnetzwerks mit allen anderen Knoten des Gesamtnetzwerks/eines anderen Teilnetzwerks verbunden sind.

Um sich über die Struktur der Kommunikationsbeziehungen hinaus der spezifischen diskursiven Deutung des Protestgeschehens in den Teilnetzwerken und deren Veränderung im

\footnotetext{
${ }^{12}$ In absoluten Zahlen ausgedrückt umfasst Netzwerkmodell 1 (Prolog) 96 Knoten mit 246 Kanten; der Grad-Wertebereich beträgt 13 bis 239 Verbindungen. Netzwerkmodell 2 (Solidarisierung) umfasst 480 Knoten mit 5661 Kanten; der Grad-Wertebereich beträgt 26 bis 847 Verbindungen. Netzwerkmodell 3 (Eskalation) umfasst 1396 Knoten mit 17853 Kanten; der GradWertebereich beträgt 22 bis 3981 Verbindungen. Netzwerkmodell 4 (Schock) umfasst 1410 Knoten mit 20141 Kanten; der GradWertebereich beträgt 25 bis 3752 Verbindungen. Netzwerkmodell 5 (Epilog) umfasst 860 Knoten mit 11566 Kanten; der GradWertebereich beträgt 30 bis 1475 Verbindungen.
} 
Zeitverlauf anzunähern, wurde im Anschluss an die Netzwerkanalyse eine Auszählung der in den (Re-)Tweets enthaltenden Schlüsselwörter durchgeführt, das heißt von Wörtern, die besonders häufig auftreten und eine entsprechend hohe Aussagekraft besitzen. Den Textkorpus bildeten alle (Re-)Tweets der im Netzwerkmodell enthaltenen NutzerInnen, deren Textinhalt zunächst einem mehrstufigen Säuberungsverfahren mit dem Ziel der Informationsrückgewinnung unterzogen wurde. Dazu wurde der Textkorpus in Kleinschreibung überführt, bevor Satzund Sonderzeichen sowie sogenannte Stoppwörter, also sehr häufig auftretende Wörter ohne größere semantische Relevanz (beispielsweise Artikel und Präpositionen, aber auch unverhältnismäßig häufig verwendete Wörter wie „G20“ und „Hamburg“) aus dem Text entfernt wurden. Anschließend wurden die Wörter einer sogenannten Lemmatisierung unterzogen, bei der sie in ihre Grundform überführt werden. Einen wichtigen Schritt stellt hierbei das sogenannte stemming dar, in dessen Verlauf Wörter auf ihren Wortstamm reduziert werden. ${ }^{13}$ Auf Basis des so aufbereiteten Textkorpus' wurden separierte Schlüsselwort-Auszählungen für alle fünf Episoden sowie für jedes der Teilnetzwerke durchgeführt (siehe Abbildungen 6-10 im Appendix).

Um die semantische Funktion der so identifizierten Schlüsselwörter für den Teilnetzwerkspezifischen Diskurs analysieren zu können, wurde die quantitative Textanalyse mit einer qualitativen Auswertung systematisch ausgewählter Tweets kombiniert. Die Auswahl orientiert sich dabei an den am häufigsten weitergeleiteten Tweets der zehn NutzerInnen mit dem höchsten gewichteten Eingangsgrad pro Episode und Teilnetzwerk. Insgesamt wurden auf diese Weise 90 Tweets gesichtet und ausgewertet, die als besonders charakteristisch für den TwitterDiskurs in bestimmten Zeitabschnitten erachtet werden können (siehe Dokumentation Textinhalt und Retweet im Appendix). Dabei wurde gezielt die semantische Einbettung der Schlüsselwörter in den Aussagen der Twitter-NutzerInnen betrachtet. Unter Rückbezug auf die Ergebnisse der Netzwerkanalyse können so zum einen Verschiebungen des Diskurses im Zeitverlauf sowie zum anderen Divergenzen in der Deutung des Geschehens dargestellt werden. Die zentralen Ergebnisse dieses triangulierten Verfahrens der Textanalyse werden im folgenden Abschnitt dargestellt.

\section{Ergebnisse: die Homogenisierung und Polarisierung des Twitter-Diskurses entlang der Ge- waltfrage}

Die Netzwerkanalyse zeigt, dass die Diskursarena Twitter im Zuge des Protestgeschehens immer stärker in zwei voneinander getrennte Teilnetzwerke zerfällt. Damit einher geht eine deutliche Ausweitung des gesamten Diskurses im Zeitverlauf. Dieser Prozess wird hier als eine voranschreitende Polarisierung der Kommunikationskreise gedeutet (Burger/Luginbühl 2014: 18). Während das Netzwerkmodell in Episode 1 (Prolog) durch eine geringe Anzahl an Knoten und eine verhältnismäßige hohe Dichte gekennzeichnet ist (d.h. das gesamte Netzwerk bildet eine geschlossene Gemeinschaft ohne Teilnetzwerke), so deutet sich bereits ab Episode 2 (Solidarisierung) die Entstehung eines Gegendiskurses an, was besonders daran zu erkennen ist, dass

\footnotetext{
${ }^{13}$ So werden bspw. die Wörter Journalist [en], Journalistin[nen], journalistisch[e/en/er/es] auf ihren Wortstamm journalist reduziert.
} 
sich ein zunächst noch kleines, im weiteren Zeitverlauf aber stark anwachsendes Teilnetzwerk formiert. Dieses Teilnetzwerk ist in den Abbildungen in einem dunklen Grauton dargestellt und wird im Folgenden als Teilnetzwerk B bezeichnet. Parallel hierzu ist zu beobachten, dass das in hellem Grauton dargestellte Teilnetzwerk (im Folgenden Teilnetzwerk A genannt) im Vergleich zu Episode 1 massiv anwächst. Dass es sich bei dem für Episode 1 modellierten (Gesamt-)Netzwerk und dem Teilnetzwerk A der zweiten Episode tatsächlich um Momentaufnahmen einer kontinuierlichen Ausweitung „desselben“ Netzwerks handelt, zeigt sich auch daran, dass die zentralen Positionen innerhalb der (Teil-)Netzwerke von denselben NutzerInnen besetzt sind. Entsprechende Beobachtungen wurden für beide Teilnetzwerke im Übergang aller Episoden gemacht. Dies deutet darauf hin, dass es in beiden Teilnetzwerken eine überschaubare Anzahl an NutzerInnen gibt, die als mehr oder weniger konstante Hubs (Knoten mit besonders vielen Verbindungen) die Teilnetzwerke zusammenhalten und über den gesamten Untersuchungszeitraum hinweg einen kontinuierlichen Einfluss auf den Diskurs ausüben. Zu diesen zentralen NutzerInnen gehören im Fall des Teilnetzwerks A vor allem die (primär journalistischen) Profile der NutzerInnen ndaktuell ${ }^{14}$, dpa, ErikMarquart, MatthiasMeisner, martinkaul, metronaut, $n d r$, PerspektiveOn, SPIEGELONLINE und tagesschau, die in zwei oder mehr der fünf untersuchten Episoden zu den NutzerInnen mit den zehn höchsten gewichteten Eingangsgraden zählen. Als kontinuierlich relevante Hubs mit zwei oder mehr Top-Ten-Positionierungen innerhalb des Teilnetzwerks B fungieren die (privaten) NutzerInnen DoraBromberger ${ }^{15}$, Hartes_Geld, krk979, lawyerberlin, OssiMarzahn76, ShakRiet und Varg_I_Veum sowie das Profil der Deutschen Polizeigewerkschaft Berlin DpolGBerlin.

Bezüglich der Beziehungen der Teilnetzwerke zueinander fällt auf, dass in Episode 2 noch eine verhältnismäßig hohe Dichte zwischen den Teilnetzwerken besteht, was auf ein vergleichsweise häufiges Weiterleiten von Tweets aus dem jeweils anderen Teilnetzwerk hindeutet. Diese Form des buchstäblichen Teilens von Positionen des Gegendiskurses lässt sich im weiteren Zeitverlauf immer weniger beobachten, wie an der abnehmenden Dichte der Verbindungen von Teilnetzwerk A nach Teilnetzwerk B und der entgegengesetzt ausgerichteten Verbindungen zu erkennen ist. Dieser von uns als zunehmende strukturelle Schließung der Teilnetzwerke gedeutete Prozess drückt sich auch in der räumlichen Konstruktion der Netzwerkmodelle aus. Insbesondere ein Vergleich der Modelle der Episoden 3, 4 und 5 (Eskalation, Schock und Epilog) zeigt, dass die Teilnetzwerke zunehmend auseinanderdriften und die Zahl der zwischen ihnen bestehenden Verbindungen deutlich zurückgeht. Dieses als Polarisierung der Diskursarena gedeutete Auseinanderdriften der Teilnetzwerke spiegelt sich in der quantitativen wie qualitativen Auswertung des Textinhalts der (Re-)Tweets wider, deren Ergebnisse wir im Folgenden in kondensierter Form präsentieren. Dabei werden die Beobachtungen beider Analyseschritte mit relevanten Ereignissen aus dem Untersuchungszeitraum in Beziehung gesetzt, um so das Diskursgeschehen hermeneutisch besser durchdringen zu können.

\footnotetext{
${ }^{14}$ Bei den hier verwendeten Bezeichnungen der Twitter-NutzerInnen handelt es sich um die sogenannten screen names, d.h. um Namenskürzel, die gezwungenermaßen aus einem einzigen Wort bestehen. Jedem screen name entspricht dabei ein (meist längerer) NutzerInnen-Name (im Fall von ndaktuell bspw. neues deutschland).

${ }^{15}$ Bei dem/der hier beschriebenen NutzerIn handelt es sich um ein mittlerweile aufgrund von Verstößen gegen die Nutzerrichtlinien gesperrtes Twitter-Profil. Bei dem/der derzeit (Stand Oktober 2019) aktiven NutzerIn mit demselben screen name handelt es sich um ein anderes Profil.
} 
Die als besonders relevant identifizierten Schlüsselwörter der ersten, von einem singulären Netzwerk beschriebenen Episode deuten auf einen Diskurs hin, der sich primär um die Frage nach der (gerichtlichen) Zulassung von Protestcamps im Hamburger Stadtraum (protestcamp/camp/verboten/erlaubt) sowie um die vermeintlich repressive Praxis der Hamburger Polizei dreht (polizei/polizist/anwalt/notdien). Dabei zeigt die qualitative Auswertung der Tweets, dass insbesondere die Gefahrenprognose und das vermeintlich unverhältnismäßige Kräfteaufgebot der Polizei zentrale Themen darstellen.

RT @ndaktuell: \#G20-Festung Hamburg in Zahlen: 19000 Polizisten, 3000 Fahrzeuge, 11 Hubschrauber, 185 Hunde, 70 Pferde \#NoG20 https://t.co/8vh8TmBp85 $(E 1.1)^{16}$

RT@MDRAktuell: Streit um G20-\#Protest: Hamburger Polizei will auch nach BVG-Urteil Camp im Park nicht dulden. "Unter Sicherheitsaspekten nicht vertret$\operatorname{bar}^{\prime \prime}(E 1.3)$

RT@fozzybear77: Anwalts-Notdienst zum \#G20-Gipfel: "Die Polizei betreibt Stimmungsmache" https://t.co/BCOB8kPzkB \#noG20 (E1.6)

Aufgrund der Antizipation der bevorstehen Ereignisse, die in den Tweets zum Ausdruck kommt, wurde Episode 1 als Prolog bezeichnet.

Der insgesamt als protestnah zu charakterisierende Diskurs der ersten Episode findet seine Entsprechung im Diskurs des Teilnetzwerks A der Episode 2, der von einer Skandalisierung der Räumung des Protestcamps im Elbpark Entenwerder (polizei/camp/zelt/protestcamp) sowie eine daran anknüpfende Auseinandersetzung um die vermeintliche Missachtung demokratischer Grundrechte geprägt ist (demokrati).

RT@ndaktuell: Das ist ein »Putsch der \#Polizei gegen die Justiz«, so der Anwalt des @Antikap_Camp. https://t.co/mbZplghn9Y \#NoG20 \#Entenwerder \#G20 (E2.1)

$R T$ @taznord: Sieht aus wie in Russland: Ein gepfefferter Kommentar von Patricia Hecht zum Polizeieinsatz in Hamburg \#NoG20 \#G20HH https://t.co/wP8SXAP3Y7 (E2.6)

Der sich in Episode 2 bereits langsam formierende Gegendiskurs des Teilnetzwerks B bedient sich einer ähnlichen Themensetzung, ist jedoch von konträren Positionen geprägt, die insbesondere auf die Delegitimierung der Proteste als gewalttätig oder extremistisch abzielen (demonstrant/protestwell/protestcamp/gewaltbereit/linksextremist/gewalt). Dabei wird unter anderem auf Einschätzungen des Verfassungsschutzes Hamburg und des Bundesinnenministers Bezug genommen.

RT @heutejournal: Hamburgs \#Verfassungsschutz warnt vor 650 gewaltbereiten \#Linksextremisten. 3 Gruppen sind bei den Anti-\#G20-Demos im Fokus der Ermittler: $\underline{\text { https://t.co/e61SsLeMkc }}($ E2.11)

\footnotetext{
${ }^{16}$ Die Tweets werden hier in ihrer Gänze wiedergegeben, das heißt inklusive des Re-Tweet-Kürzels RT, des mit @ gekennzeichneten Verweises auf den Originalverfasser des Tweets, möglicherweise verwendeter Hashtags sowie Verlinkungen anderer Twitter-Inhalte.
} 
RT@BMI_Bund: \#deMaizière: "Gewalttätige Demonstrationen stehen nicht unter dem Schutz des GG." \#Linksextremismus \#Verfassungsschutzbericht \#G20 https://t.co/7ifDiB9pZH (E2.20)

RT@SoliDavidwache: Allein das Wort "Demonstranten" ist falsch. Wer Gewalt ausübt steht nicht unter dem Schutz der Versammlungsfreiheit \#G20HAM17 \#Polizei https://t.co/j1eB5Xp20y (E2.13)

Trotz des sich formierenden Gegendiskurses wurde Episode 2 aufgrund der überwiegenden Anzahl an positiven Bezugnahmen auf die von der Räumung betroffenen Protestierenden als Phase der breiten Solidarisierung mit den Protestakteuren bezeichnet.

In Episode 3 zeigt sich ein deutlich ausgewogeneres Bild mit zwei annähernd gleich starken Teildiskursen. Dabei verhärten sich die konträren Positionen zunehmend, wobei insbesondere die Gewaltfrage und die Praxis der Polizei entscheidende Streitpunkte bilden. Zentrale Ereignisse für den Diskurs des protestnahen Teilnetzwerks A sind dabei die gewaltsame Auflösung der Demonstration Welcome to Hell (polizei/prot/gewalt/demo) sowie der Entzug der Akkreditierung von JournalistInnen durch die Polizei (akkreditier/journalist/entzog). Dabei dominiert bezüglich der Welcome to Hell-Demonstration die Auffassung, die Polizei habe die Lage gezielt eskalieren lassen. Bezüglich des Entzugs der Akkreditierungen wird eine kritische Haltung eingenommen. Ferner wird die Gewaltausübung seitens der Polizei kritisiert.

RT@NiemaMovassat: Von wem ging Gewalt bei den Protesten gegen \#G20 aus? Viele Journalisten haben eine klare Antwort: Die Polizei eskalierte. \#welcometohell https://t.co/ocSikcabsj (E3.10)

RT@Der_Postillon:Zur Deeskalation: Hamburger Polizei fährt Atomrakete auf \#G20 \#G20HH17 https://t.co/tCOgefk7Hd (E3.3)

RT@ martinkaul: Lange Liste: Din-A-4 voller Namen haben diese Polizisten am Pressezentrum in der Hand. Wer drauf steht, bekommt Akkreditierung entzogen \#G20 https://t.co/REqLQ4GZAm (E3.1)

RT@PerspektiveOn: Presse Kollege von uns aus FFM wurde von @PolizeiHamburg der Arm gebrochen. Jetzt im Krankenhaus \#G20 \#NoG20 \#BlockG20 \#WelcomeToHell (E3.4)

In dem eher protestfeindlich eingestellten Teilnetzwerk B werden abermals konträre Positionen bezogen. Hier wird die Verantwortung für die Eskalation der Welcome to Hell-Demonstration bei den Protestierenden gesucht, die durch einen sogenannten Schwarzen Block verübten Brandanschläge auf parkende Autos in Altona und die Plünderungen im Schanzenviertel kritisiert (gewalt/link/autos/schwarz/block/krawall) sowie Berichte über vermeintlich schwer verletzte PolizistInnen verbreitet (polizei/polizist/verletzt). Dabei wird mangelnde Berichterstattung beklagt und versucht, linke Politik insgesamt zu diskreditieren.

RT@DpolGBerlin: Liebe @tagesschau, es sind ebenfalls Kolleginnen \& Kollegen zum Teil schwer verletzt worden! Keine Berichterstattung wert? Unfassbar! \#G20 https://t.co/pSHkjhWUi3 (E3.12) 
RT@ShakRiet: Feindsender Ost: Leider weigern sich die deutschen Medien, die Plünderungen in der \#Schanze zu dokumentieren. \#G20 https://t.co/E1anGfc3rX (E3.13)

RT @lawyerberlin: Hallo Herr Justizminister \#Maas: Wo bleibt Ihr klares Statement gegen linksextremistische Gewalt? \#G20HAM \#G20HH2017 \#G20Hamburg \#G20Summit https://t.co/rXdKboAneL (E3.19)

RT@Steinhoefel: G20/Hamburg zeigt, wie wichtig es war, Millionen im "Kampf gegen rechts" investiert zu haben. (E3.16)

Aufgrund der ab Episode 3 eintretenden strukturellen Schließung der Teildiskurse bei gleichzeitiger Verhärtung der eigenen Positionierung zur Gewaltfrage wurde Episode 3 als Eskalation bezeichnet.

In Episode 4 setzt sich die Auseinandersetzung um die Gewaltfrage fort, wobei hier auch im protestnahen Teilnetzwerk A kritische Positionen bezüglich linker Militanz bezogen werden. Im Vordergrund steht dabei die Frage nach der Sinnhaftigkeit von gewaltsamem Protest (friedlich/gewalt/prot/krawall); als Verantwortliche für die Ausschreitungen werden jedoch insbesondere die Polizei und Hamburgs Erster Bürgermeister benannt.

RT@ErikMarquart: Einige Vermummte haben Apple-Store geplündert und sich dann um die iPhones gestritten. Das ist nicht links, sondern beknackt. \#schanze \#g20 https://t.co/luA2t7DfiD (E4.1)

RT@ndr: Warum nicht in Blankenese oder in Pöseldorf? Rote Flora-Anwalt-Andreas Beuth hat kein Verständnis für die Randale im eigenen Viertel. \#G20 https://t.co/NGp9be2fpm (E4.2)

RT@PatrickGensing: Polizei bei \#G20: Polizei bei \#G20: Eskalieren, als nichts los war - überfordert, als was los war. Gipfel wurde geschützt, Bürger nicht. Polit. Verantwortung? (E4.10)

RT@extra3: Die Fehleinschätzung des Jahres kommt von Hamburgs Bürgermeister Olaf Scholz. $\left({ }^{*}\right.$ slow clap ${ }^{*}$ \#G20HAM17 \#welcometohell \#schanze \#g20 https://t.co/bCLecPiKXX (E4.6)

Das protestfeindliche Teilnetzwerk B führt währenddessen einen mit Episode 3 vergleichbaren Diskurs (abermals dominieren hier die Schlüsselwörter link/krawall/gewalt/schwarz), wobei eine zunehmende Verknüpfung mit (extrem) rechten Positionen und einer Kritik an politisch linken Parteien zu beobachten ist, denen eine radikalisierende Wirkung auf linke GewalttäterInnen zugeschrieben wird. Daran anknüpfend werden stellenweise offen Sympathien für Gewalt gegen Demonstrierende zum Ausdruck gebracht.

RT @Varg_I_Veum: \#KölnHbf hatte nichts mit Zuwanderung zu tun \#Breitscheidplatz hatte nichts mit dem Islam zu tun \#G20 hatte nichts mit Linken zu tun. Aha. (E4.15) $R T @ l a w y e r b e r l i n:$ Machen wir uns nichts vor: Die linksextremistischen Verbrecher aus \#Hamburg werden von \#Linken und \#SPD als Vorfeldorganisation gedeckt. https://t.co/i65KqF3vug (E4.13) 
RT @dushanwegner: Sie schaffen seit Jahren den geistigen Boden für diese Täter. Jetzt heucheln Sie nicht. https://t.co/fwymZAeAj3 (E4.11)

RT@Hartes_Geld: Bei diesem Gerangel hat der Polizist dem Demonstrierenden wohl klar gemacht, was \#WelcomeToHell bedeutet. :) \#G20HH2017 \#G20 https://t.co/Hvqn $3 v F O T V$ (E4.16)

Aufgrund der in beiden Teilnetzwerken vorherrschenden Bestürzung über die (Gewalt-)Ereignisse wurde Episode 4 als Schock bezeichnet.

In Episode 5 werden schließlich die diskursiven Kondensate der Protestwoche erkennbar (daher die Bezeichnung Epilog). Hier erreicht die Polarisierung des Diskurses ihren Höhepunkt; die in den Teilnetzwerken vertretenen Positionen scheinen kaum noch Austausch zuzulassen. Im protestnahen Teilnetzwerk A wird die von verschiedener Seite ausgeübte Gewalt der Protestwoche aufgearbeitet (polizei/krawall/link/schwarz/polizeigewalt). Dabei wird die Polizeigewalt als zentraler Faktor für die Eskalation der Ereignisse hervorgehoben.

RT@ErikMarquardt: Man hilft weder der Polizei noch dem Vertrauen in den Rechtsstaat, wenn man so tut als wenn es bei G20 null Polizeigewalt gab. \#maischberger (E5.3)

Auch wird die Reaktion der Politik und hier insbesondere die angekündigten Entschädigungszahlungen sowie die Forderung nach einer Verschärfung der Polizeigesetzgebung als unverhältnismäßig dargestellt.

RT @KatharinaKoenig: Für Geschädigte \#G20-Gipfel (angezündete Läden / Autos) gibt \#Hamburg 10 Millionen €.Für Angehörige \#NSU-Opfer ఓamp; Betroffene

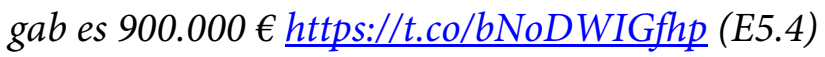

RT @kattascha: Wer nach den Beschlüssen der letzten Jahren Grundrechte weiter abbauen will, hat jedes Maß verloren. Karlsruhe erstickt unter Klagen. \#G20 https://t.co/1KevUcyqRc (E5.5)

Darüber hinaus werden vermeintliche Grundrechtsverletzungen und hier insbesondere der Entzug von Akkreditierungen sowie der sich im Kontext der G20-Proteste formierende politisch rechte Gegendiskurs thematisiert.

RT@PengBerlin: Leude es is soweit: reicht alles ein was \#G20 Grundrechtsverletzungen dokumentiert - die neue Webseite is online! https://t.co/LPc58H31HH https://t.co/J4YtLt7Q2b (E5.7)

RT@tagesschau: Journalisten auf "Schwarzer Liste" bei G20 https://t.co/odnMPn79Q2 \#G20 \#Jourrnalisten (E5.6)

RT@ndaktuell: Gefährlicher als Randale: @Linksdings über einen Backlash gegen alles Linke \&amp; den rasenden Zug ins Autoritäre https://t.co/bQMJVKIpfU \#G20 (E5.2) 
Im protestfeindlichen Teilnetzwerk B lässt sich währenddessen eine Verhärtung der linksfeindlichen Positionen beobachten, die den Diskurs maßgeblich prägen (link/krawall/gewalt/schwarz/block/straftat). Zentrale Motive sind der Vergleich der Proteste mit rechtem politischem Aktivismus, Terrorismusvorwürfe gegen linke Organisationen und Gruppen sowie primär gegenüber der Bundeskanzlerin vorgebrachte Rücktrittsforderungen.

RT @lawyerberlin: Seit 40 Jahren wird der Begriff rechts diskreditiert und verfälscht. Seit \#G20 ist klar: \#Links bedeutet Gewalt und Intoleranz.Danke \#Antifa (E5.17)

RT@Hartes_Geld: Ich finde die Presse sollte diesen altgedienten Anti-Faschisten und \#G20-Veteranen sofort als "Pegida-Wut-Bürger" verkaufen.

https://t.co/Pcg7iK8Prx (R5.12)

$R T @ D r D a v i d B e r g e r: D i e ~ A n t i f a$ muss endlich als terroristische Vereinigung eingestuft werden! https://t.co/0X6BhAlMdd \#Antifa \#Verfassungsschutz (E5.15)

RT@Varg_I_Veum: \#KölnHbf: Keine Rücktritte \#Breitscheidplatz: Keine Rücktritte \#G20: Keine Rücktritte Niemand übernimmt in D Verantwortung für irgendwas.

RT@SteinbachErika: Die Kanzlerin wollte Hamburg. Sie trägt die Verantwortung Jeder Kenner der linksradikalen Szene wußte, dass Hamburg denkbar ungeeignet ist. https://t.co/rIVvorrGww (E5.16)

Zusammenfassend zeigt die Triangulation aus quantitativer und inhaltlicher Textanalyse, dass sich die Teilnetzwerke in ihrer normativen Bewertung der Ereignisse deutlich voneinander unterscheiden und sich zudem mit fortschreitendem Verlauf kaum noch aufeinander beziehen. Die Diskursteilnehmenden richten sich stattdessen immer mehr in einer Art digitalen Echokammer ein, in der jeweils völlig konträre Bewertungen der Ereignisse über die ungefilterte Redistribution von Tweets generiert werden. Aus diesem Grund kann einerseits von einer Homogenisierung des Diskurses innerhalb jener Kommunikationskreise gesprochen werden, aus denen die Teilnetzwerke bestehen. Andererseits kann parallel zur Homogenisierung der jeweiligen Teildiskurse eine starke Polarisierung des Gesamtdiskurses beobachtet werden, da die Gewaltfrage innerhalb der Teilnetzwerke diametral entgegengesetzt diskutiert und normativ bewertet wird: Während das Teilnetzwerk B die Gewaltfrage vergleichsweise einhellig im Sinne der staatlichen Ordnungsmacht auslegt und die Polizeigewalt als legitime Gegengewalt rahmt, zeigt sich das Teilnetzwerk A diesbezüglich eher heterogen und teilweise durchaus kritisch in den Bewertungen der Gewalt, die von Protestierenden ausgeht; gleichwohl wird hier die Eskalation des Protestgeschehens weit überwiegend als Resultat der polizeilichen Interventionen gedeutet. Wie der Vergleich der Netzwerke über die verschiedenen Episoden der Protestwoche hinweg zudem deutlich macht, bildet sich das protestfeindliche Teilnetzwerk B erst im Zuge der Eskalation des Geschehens, während die Protestseite von Beginn an eine stabile Struktur an Kommunikationskreisen aufweist. Dies deutet darauf hin, dass es sich auf protestfeindlicher Seite um einen Prozess der Solidarisierung von außen handelt, bei dem zuvor entweder gar nicht oder kaum in den hier betrachteten Twitter-Diskurs eingebundene Akteure sich zunehmend in den Diskurs einbringen, um nicht nur die Gewalt der Protestierenden, sondern gleichsam den gesamten Protest zu diskreditieren und der Polizei den Rücken zu stärken. Auf Seiten der Protestierenden wiederum ist die zunehmende Polarisierung des Twitter-Diskurses einem 
Solidarisierungseffekt nach innen geschuldet, der durch die hier zu beobachtende Formierung der Gegenseite befördert wird. Denn die schrittweise Verschiebung des Diskurses hin zur Gewaltfrage hat nicht zuletzt zur Folge, dass das gesamte Protestspektrum zunehmend unter Legitimationsdruck gerät.

\section{Schlussbetrachtung}

Die eingangs erwähnte, nur schwer zu schließende Lücke zwischen Diskurs und Praxis oder diskursiven Ereignissen einerseits und konkreten Gewalthandlungen andererseits bleibt auch im Anschluss an das hier angewandte Verfahren ein grundlegendes methodisches Problem. Insbesondere Arbeiten, die auf empirischem Material aus sozialen Medien basieren, sehen sich diesbezüglich vor besondere Herausforderungen gestellt, zumal der Gebrauch moderner Kommunikationsmedien aus dem Bereich der Protestforschung kaum noch wegzudenken ist. Die fragliche Lücke zu schließen, war nicht unser Anspruch, da das empirische Material ausschließlich auf der Ebene medialer Diskurse zu situieren ist. Gleichwohl eröffnet die hier angewandte Methode Möglichkeiten, die diskursiven Dynamiken in einen direkten Zusammenhang mit den situativen Dynamiken des Protestgeschehens zu bringen - und zwar nicht nur, da diese zeitlich mehr oder weniger zusammenfallen. Vielmehr begreifen wir das Weiterleiten von Tweets in Form von Re-Tweets als eine kommunikative Praxis, die schon als solche nicht mehr bloß diskursive Ereignisse hervorbringt, sondern unter Umständen ein besonderes Mobilisierungspotenzial zu entfalten vermag. Genau dieses Potenzial lässt sich am hier untersuchten Fall exemplarisch darstellen und mit der Einsicht verknüpfen, dass es sich um eine stark polarisierende soziale Mobilisierung handelt, die zur Eskalation des Protestgeschehens beiträgt.

Das Versenden von Re-Tweets stellt aus unserer Sicht keinen rein technisch-operativen Akt dar. Vielmehr muss das Re-Tweeten von Nachrichten zugleich als kommunikative Praxis begriffen werden, durch die soziale Beziehungen zu anderen DiskursteilnehmerInnen aktiv hergestellt werden: „Spreading tweets is not simply to get messages out to new audiences, but also to validate and engage with others" (Boyd et al. 2010: 1). Die Grundannahme, an der sich unsere Analysestrategie orientiert, lautet hieran anschließend, dass es weit überwiegend affirmative Haltungen zum ursprünglichen Tweet-Inhalt sind, die das Weiterleiten einer Nachricht motiviert - eine Einschätzung, zu der auch Forschungsbeiträge kommen, die sich explizit mit dieser Frage beschäftigen: „[R]etweeting indicates not only interest in a message, but also trust in the message and the originator, and agreement with the message contents" (Metaxas at al. 2014: 1). ${ }^{17}$ Die Praxis des Re-Tweetens stellt aus dieser Perspektive Beziehungen zwischen unterschiedlichen DiskursteilnehmerInnen her, die sich zu vergleichsweise geschlossenen Kommunikationskreisen verbinden können. Diese Form der strukturellen Schließung kommunikativer Beziehungen geht im hier untersuchten Fall des Twitter-Diskurses während der G20-Proteste

\footnotetext{
${ }^{17}$ Zusätzliche Plausibilität gewinnt diese von uns zugrunde gelegte heuristische Annahme dadurch, dass ausschließlich unkommentierte Re-Tweets zur Rekonstruktion der Netzwerke herangezogen wurden. Dass SenderInnen einer Nachricht dem darin enthaltenen Inhalt ablehnend gegenüberstehen, diesen zugleich aber unkommentiert an die eigenen Follower weiterleitet, ist äußerst unwahrscheinlich, zumal die Empfänger den Re-Tweet in diesem Fall nur sehr bedingt als Ablehnung erkennen können.
} 
mit einer kognitiven Schließung einher, die zwei diametral entgegengesetzte normative Standpunkte bezüglich der Gewaltfrage generiert.

Wie unsere empirische Untersuchung zeigt, entfalten die in Twitter generierten Kommunikationsbeziehungen mit fortschreitendem Protestverlauf einen selbstverstärkenden Polarisierungsprozess, bei dem die inhaltliche Debatte und die Konfrontation mit abweichenden Positionen einer selbstbezüglichen Kommunikation weicht, die fast nur noch um die Gewaltfrage kreist und dabei den jeweils eigenen normativen Standpunkt radikalisiert. Dieser Zusammenhang aus struktureller und kognitiver Schließung transformiert zumindest temporär die Wahrnehmungs- und Deutungsmuster der Akteure derart, dass eine diskursive Dynamik entsteht, die quasi nur noch Freund-Feind-Unterscheidungen zulässt: Eine kritische Distanzierung zur Gewalt auf der Protestseite ist somit kaum noch möglich, ohne damit gleichzeitig dem gesamten Protestspektrum in den Rücken zu fallen; ebenso wenig kann die polizeiliche Gewalt kritisiert werden, ohne zugleich zu SympathisantInnen von GewalttäterInnen erklärt zu werden. Spitzen sich Prozesse der Polarisierung und damit einhergehend der Homogenisierung von gewaltlegitimierenden Diskursen derart zu, öffnen sich Wege zur Entgrenzung von Gewalt, auch weil sich gleichzeitig die Wege zu ihrer diskursiven Einhegung verschließen. ${ }^{18}$ In der Rekonstruktion und Analyse der Twitter-Netzwerke zeigt sich damit einmal mehr die Bedeutung von endogen verursachten Dynamiken der Konflikteskalation, die erst im Zuge des Protestverlaufs selbst entstehen und denen eine tragende Rolle bei der Emergenz von Gewaltmassen zukommt, wie sie im Fall der Hamburger Protestwoche zu beobachten war.

\section{Danksagung}

Die Autoren bedanken sich bei den externen Gutachterinnen und Gutachtern und den Redaktorinnen und Redaktoren des Journals sozialpolitik.ch für die wertvollen Hinweise und Kommentare.

\section{Deklaration von Interessenkonflikten}

Die Autoren deklarieren keine Interessenkonflikte in Bezug auf Forschung, Autorinnenschaft und Publikation des Artikels.

\section{Finanzierung}

Die Autoren bedanken sich bei der Wirtschafts- und Sozialwissenschaftlichen Fakultät der Universität Potsdam für die finanzielle Unterstützung, ohne die weder die Forschung zu diesem Artikel noch eine Mitarbeit an dem Projekt Mapping \#NoG20 möglich gewesen wären.

\footnotetext{
${ }^{18}$ Mit Blick auf die verfassungsrechtlich höchst umstrittene Praxis der behördlichen Strafverfolgung im Anschluss an die Gipfelproteste lässt sich diese These auch weit über die Protestwoche hinaus für Formen staatlicher Gewalt formulieren. Aufschlussreich dafür ist unter anderem die Erkenntnis, dass sich die hier beschriebene Polarisierung der Standpunkte im Anschluss an die Protestwoche nicht etwa abschwächt, sondern weiter verhärtet, wie ein Blick auf die Netzwerke der Episoden Schock (Abb. 4 Appendix) und Epilog (Abb. 5 Appendix) nahelegt.
} 


\section{Literaturverzeichnis}

Bastian M., Sebastien Heymann und Mathieu Jacomy (2009). Gephi: an open source software for exploring and manipulating networks. Conference Paper: International AAAI Conference on Weblogs and Social Media. San José.

Blondel, Vincent D., Renaud Lambiotte, Jean-Loup Guillaume und Etienne Lefebvre (2008). Fast unfolding of communities in large networks. Journal of Statistical Mechanics: Theory and Experiment, 2008 (10), DOI: 10.1088/1742-5468/2008/10/P10008.

Bosi, Lorenzo, Charles Demetriou und Stefan Malthaner (2014). Introduction: A Contentious Politics Approach to the Explanation of Radicalization. In: Lorenzo Bosi, Charles Demetriou und Stefan Malthaner (Hg.). Dynamics of Political Violence: A Process-Oriented Perspective on Radicalisation and the Escalation of Political Conflict. Farnham: Ashgate, 2-23.

Bosi, Lorenzo, und Donatella Della Porta (2012). Micro-mobilization into Armed Groups: The Ideological, Instrumental and Solidaristic Paths. Qualitative Sociology 35(4), 361-381.

Boyd, Danah, Scott Golder und Gilad Lotan (2010). Tweet, Tweet, Retweet: Conversational Aspects of Retweeting on Twitter. Proceedings of 43rd Hawaii International Conference on System Sciences, 110.

Buechler, Steven M. (2004). The Strange Career of Strain and Breakdown Theories of Collective Action. In: David A. Snow, Sarah A. Soule und Hanspeter Kriesi (Hg.). The Blackwell Companion to Social Movements. Oxford: Blackwell, 47-66.

Burger, Harald und Martin Luginbuhl (2014). Mediensprache. Eine Einführung in Sprache und Kommunikationsformen der Massenmedien. Berlin: De Gruyter Studium.

Collins, Randall (2008). Violence: A Micro-sociological Theory. Princeton: Princeton University Press.

Dang-Anh, Mark (2019). Protest twittern: Eine medienlinguistische Untersuchung von Straßenprotesten. Bielefeld: transcript.

Della Porta, Donatella (1995). Social Movements, Political Violence, and the State: A Comparative Analysis of Italy and Germany. Cambridge: Cambridge University Press.

Della Porta, Donatella (2008). Research on Social Movements and Political Violence. Qualitative Sociology 31(3), 221-230.

Della Porta, Donatella (2012). Social Movement Studies and Political Violence. In: Marie Breen-Smyth (Hg.). The Ashgate Research Companion to Political Violence. Farnham, London: Ashgate, 243-260.

Della Porta, Donatella (2013). Clandestine Political Violence. Cambridge: Cambridge University Press.

Della Porta, Donatella und Sidney Tarrow (1986). Unwanted Children. Political Violence and the Cycle of Protest in Italy 1966-1973. European Journal of Political Research 14(5-6), 607-632.

Della Porta, Donatella, und Gary LaFree (2012). Guest Editorial: Processes of Radicalisation and DeRadicalisation. International Journal of Conflict and Violence 6(1), 4-10.

Deutschlandfunk (2017). G20-Krawalle. Wo sind die Täter? Zugriff am 03.06.20 auf https://www.deutschlandfunk.de/g20-krawalle-wo-sind-die-taeter.2852.de.html?dram:article $\mathrm{id}=390728$. 
Ebner, Julia (2019). Radikalisierungsmaschinen. Wie Extremisten die neuen Technologien nutzen und uns manipulieren. Frankfurt am Main: Suhrkamp.

Felson, Richard B. und Henry S. Steadman (1983). Situational Factors in Disputes Leading to Criminal Violence. Criminology 21(1), 59-74.

Gurr, Ted R. (1970). Why Men Rebel. Princeton: Princeton University Press.

Hartmann, Eddie (2016). Symbolic Boundaries and Collective Violence. A New Theoretical Argument for an Explanatory Sociology of Collective Violent Action. Journal for the Theory of Social Behaviour 46(2), 165-186.

Hartmann, Eddie (2017). In the Zone of Spoiled Civil Identity. The Riots in Suburban France in 2005. In: Jürgen Mackert und Bryan S. Turner (Hg.). The Transformation of Citizenship, Vol. 3., Struggle, Resistance, and Violence. London, Routledge, 39-55.

Hartmann, Eddie (2019a). Produktiver Reduktionismus. Randall Collins' Mikrosoziologie der Gewalt. Mittelweg 36, 28(1-2), 40-59.

Hartmann, Eddie (2019b). Die Gewalttheorie von Jan Philipp Reemtsma. Programmatische Impulse für eine Allgemeine Soziologie der Gewalt. Zeitschrift für Theoretische Soziologie 6(1), 74-85.

Heitmeyer, Wilhelm (1997). Einleitung: Auf dem Weg in die desintegrierte Gesellschaft. In: Wilhelm Heitmeyer (Hg.). Was treibt die Gesellschaft auseinander? Frankfurt am Main: Suhrkamp, 9-28.

Heitmeyer, Wilhelm (Hg.) (1996). Die bedrängte Toleranz. Ethnisch-kulturelle Konflikte, religiöse Differenzen und die Gefahren politisierter Gewalt. Frankfurt am Main: Suhrkamp.

Hoebel, Thomas (2019). „Wir haben Charlie Hebdo getötet!“ Konsequenzielle Dritte und die Erklärung fortgesetzter Gewalt. Mittelweg 36, 28(1-2), 99-123.

Hoebel, Thomas, und Stefan Malthaner (2019). Über dem Zenit. Grenzen und Perspektiven der situationistischen Gewaltforschung. Mittelweg 36, 28(1-2), 3-14.

Ingold, Albert (2017). »Polizei 2.0«: Grenzen der behördlichen Öffentlichkeitsarbeit in sozialen Netzwerken. Verwaltungsarchiv 108(2), 240-265.

Katz, Jack (1988). Seductions of Crime. Moral and Sensual Attractions in Doing Evil. New York: Basic Books.

Katz, Jack (2001). From How to Why. On Luminous Description and Causal Inference in Ethnography (Part 1). Ethnography 2(4), 443-473.

Katz, Jack (2015). Epiphanie der Unsicherheit. In: Axel T. Paul und Benjamin Schwalb (Hg.). Gewaltmassen. Über Eigendynamik und Selbstorganisation kollektiver Gewalt. Hamburg: Hamburger Edition, 63-102.

Knöbl, Wolfgang (2017). Perspektiven der Gewaltforschung. Mittelweg 36, 26(3), 4-27.

Knöbl, Wolfgang (2019). Collins im Kontext. Zur Vorgeschichte der jüngeren Gewaltsoziologie. Mittelweg 36, 28(1-2), 15-39.

Koloma Beck, Teresa (2011). The Eye of the Beholder. Violence as a Social Process. International Journal of Conflict and Violence 5(2), 345-356.

Koloma Beck, Teresa (2019). Welterzeugung. Gewaltsoziologie als kritische Gesellschaftstheorie. Zeitschrift für Theoretische Soziologie 6(1),12-23. 
Lambiotte, Renaud, Jean-Charles Delvenne und Mauricio Barahona (2009). Laplacian Dynamics and Multiscale Modular Structure in Networks. Zugriff am 10.10.2019 auf https://arxiv.org/abs/0812.1770.

Malthaner, Stefan (2017). Radicalization. The evolution of an analytical paradigm. European Journal of Sociology 58(3), 369-401.

Malthaner, Stefan (2019). Riot im Schanzenviertel. Gewaltsituationen, Gelegenheitsfenster und die "Hermeneutik der Straße". Mittelweg 36, 28(1-2), 151-176.

Malthaner, Stefan, Simon Teune und Peter Ullrich (Hg.) (2018) Eskalation. Dynamiken der Gewalt im Kontext der G20-Proteste in Hamburg 2017, Berlin 2018. Zugriff am 03.06.20 auf https://g20.protestinstitut.eu.

Mayntz, Renate (1987). Eigendynamische soziale Prozesse. Anmerkungen zu einem analytischen Paradigma. Kölner Zeitschrift fü̈ Soziologie und Sozialpsychologie 39(4), 648-668.

McAdam, Doug (1982). Political Process and the Development of Black Insurgency 1930-1970. Chicago: University of Chicago Press.

McAdam, Doug, John D. McCarthy und Mayer N. Zald (Hg.) (1996). Comparative Perspectives on Social Movements. New York: Cambridge University Press.

McAdam, Doug, Sidney Tarrow und Charles Tilly (2001). Dynamics of Contention. New York: Cambridge University Press.

McCarthy, John D., und Mayer N. Zald (1977). Resource Mobilization and Social Movements: A Partial Theory. American Journal of Sociology 82(6), 1212-1241.

Metaxas, Panagiotis T., Eni Mustafaraj, Kily Wong, Laura Zeng, Megan O’Keefe und Samantha Finn (2014). Do Retweets Indicate Interest, Trust, Agreement? Zugriff am 10.10 .2019 auf https://arxiv.org/abs/1411.3555.

Morstatter, Fred, Jürgen Pfeffer und Huan Lui (2014). When is it Biased? Asessing the Rep-resentativeness of Twitter's Streaming API. Zugriff am 10.10.2019 auf https://arxiv.org/abs/1401.7909.

Morstatter, Fred, Jürgen Pfeffer, Huan Lui und Kathleen M. Carley (2013). Is the Sample Good Enough? Comparing Data from Twitter's Streaming API with Twitter's Firehose. Zugriff am 10.10.2019 auf https://arxiv.org/abs/1306.5204.

Nedelmann, Birgitta (1997). Gewaltsoziologie am Scheideweg. Die Auseinandersetzungen in der gegenwärtigen und Wege der künftigen Gewaltforschung. In: Trutz von Trotha (Hg.). Soziologie der Gewalt. Sonderheft 37 der Kölner Zeitschrift für Soziologie und Sozialpsychologie, 59-85.

Neidhardt, Friedhelm (1981). Über Zufall, Eigendynamik und Institutionalisierbarkeit absurder Prozesse. In: Von Alemann, Heine und Hans Peter Thurn (Hg.). Soziologie in weltbürgerlicher Absicht. Opladen: Westdeutscher Verlag, 243-257.

Paul, Axel T. und Benjamin Schwalb (2015). Gewaltmassen. Über Eigendynamik und Selbstorganisation kollektiver Gewalt. Hamburg: Hamburger Edition.

R Core Team (2017). R: A language and environment for statistical computing. Wien: R Foundation for Statistical Computing. Zugriff am 10.10.2019 auf https://www.R-project.org/.

Reemtsma, Jan Philipp (2006). Die Natur der Gewalt als Problem der Soziologie. Mittelweg 36, 15(5), 2 25. 
Reemtsma, Jan Philipp (2008). Vertrauen und Gewalt. Versuch über eine besondere Konstellation der Moderne. Hamburg: Hamburger Edition.

Reuter, Markus (2018). Der elektrische Türknauf und die Molotowcocktails: Falschmeldungen der Polizei auf Twitter. netzpolitik.org. Zugriff am 03.06.20 auf https://netzpolitik.org/2018/der-elektrische-tuerknauf-und-die-molotowcocktails-falschmeldungen-der-polizei-auf-twitter.

Rüdiger, Thomas-Gabriel und Petra Saskia Bayerl (Hg.) (2018). Digitale Polizeiarbeit. Herausforderungen und Chancen. Wiesbaden: Springer VS.

Schützeichel, Rainer (2019). Das Problem der Situation. Über einen operativen Begriff der Soziologie. Mittelweg 36, 28(1-2), 205-225.

Schützeichel, Rainer und Stefan Jordan (2015). Prozesse. Formen, Dynamiken, Erklärungen. Wiesbaden: Springer VS.

Sewell, William H. (1990). Collective Violence and Collective Loyalties in France: Why the French Revolution Made a Difference. Politics and Society 18(4), 527-552.

Smelser, Neil J. (1959). Social Change in the Industrial Revolution: An Application of Theory to the British Cotton Industry. Chicago: University of Chicago Press.

Sofsky, Wolfgang (1996). Traktat über die Gewalt. Frankfurt am Main: Fischer.

Sommer, Moritz und Simon Teune (2019). Sichtweisen auf Protest - Die Demonstrationen gegen den G20-Gipfel in Hamburg 2017 im Spiegel der Medienöffentlichkeit. Forschungsjournal Soziale Bewegungen 32(2), 149-162.

Sutterlüty, Ferdinand (2017). Fallstricke situationistischer Gewaltforschung. WestEnd. Neue Zeitschrift für Sozialforschung 14(2), 139-155.

Tremayne, Mark (2013). Anatomy of Protest in the Digital Era: A Network Analysis of Twitter and Occupy Wall Street. Social Movement Studies 13(1), 110-126.

Tilly, Charles (1964). The Vendée. Cambridge: Harvard University Press.

Tilly, Charles (1978). From Mobilization to Revolution. Reading: Addison-Wesley.

Tilly, Charles (2003). The Politics of Collective Violence. Cambridge: Cambridge University Press.

Tilly, Charles und Sidney Tarrow (2006). Contentious Politics. Oxford: Oxford University Press.

Tufekci, Zeynep (2017). Twitter and Tear Gas. The Power and Fragility of Networked Protest. New Haven: Yale University Press.

Von Trotha, Trutz (1997). Zur Soziologie der Gewalt. In: Trutz von Trotha (Hg.). Soziologie der Gewalt. Sonderheft 37 der Kölner Zeitschrift für Soziologie und Sozialpsychologie, 9-56.

Vosoughi, Soroush, Deb Roy and Sinan Aral (2018). The spread of true and false news online. Science 359(6380), 1146-1151.

Wickham, Hadley, Mara Averick, Jennifer Bryan, Winston Chang, Lucy D’Agostino McGowan, Romain François, Garrett Grolemund, Alex Hayes, Lionel Henry, Jim Hester, Max Kuhn, Thomas Lin Pedersen, Evan Miller, Stephan Milton Bache, Kirill Müller, Jeroen Ooms, David Robinson, Dana Paige Seidel, Vitalie Spinu, Kohske Takahashi, Davis Vaughan, Claus Wilke, Kara Woo, Hiroaki Yutani (2019). Welcome to the tidyverse. Journal of Open Source Software 4(43), 1686. 